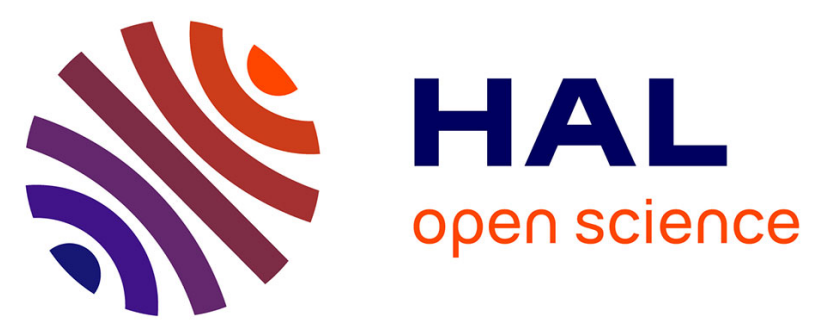

\title{
Synthesis of Polysubstituted Isoquinolines and Related Fused Pyridines from Alkenyl Boronic Esters via a Copper-Catalyzed Azidation/Aza-Wittig Condensation Sequence
}

Vankudoth Jayaram, Tailor Sridhar, Gangavaram V. M. Sharma, Fabienne Berrée, Bertrand Carboni

\section{To cite this version:}

Vankudoth Jayaram, Tailor Sridhar, Gangavaram V. M. Sharma, Fabienne Berrée, Bertrand Carboni. Synthesis of Polysubstituted Isoquinolines and Related Fused Pyridines from Alkenyl Boronic Esters via a Copper-Catalyzed Azidation/Aza-Wittig Condensation Sequence. Journal of Organic Chemistry, 2018, 83 (2), pp.843-853. 10.1021/acs.joc.7b02831 . hal-01709528

HAL Id: hal-01709528

https://hal-univ-rennes1.archives-ouvertes.fr/hal-01709528

Submitted on 27 Apr 2018

HAL is a multi-disciplinary open access archive for the deposit and dissemination of scientific research documents, whether they are published or not. The documents may come from teaching and research institutions in France or abroad, or from public or private research centers.
L'archive ouverte pluridisciplinaire HAL, est destinée au dépôt et à la diffusion de documents scientifiques de niveau recherche, publiés ou non, émanant des établissements d'enseignement et de recherche français ou étrangers, des laboratoires publics ou privés. 


\section{Synthesis of Polysubstituted Isoquinolines and Related Fused Pyridines from Alkenyl Boronic Esters via a Copper-Catalyzed Azidation/Aza-Wittig Condensation Sequence}

Vankudoth Jayaram, ${ }^{\mathrm{a}}$ Tailor Sridhar, ${ }^{\mathrm{a}}$ Gangavaram V. M. Sharma, ${ }^{\mathrm{a} *}$ Fabienne Berrée, ${ }^{\mathrm{b}}$ and Bertrand Carboni ${ }^{b *}$

a Organic and Biomolecular Chemistry Division, CSIR-Indian Institute of Chemical Technology, Tarnaka, Hyderabad-500 007, India.

${ }^{\mathrm{b}}$ Université de Rennes 1, Institut des Sciences Chimiques de Rennes, UMR CNRS 6226 Campus de Beaulieu, 35042, Rennes Cedex, France

esmvee@iict.res.in (G.V.M.S.).

bertrand.carboni@univ-rennes1.fr (B.C.).

\section{Abstract:}

An efficient and straightforward synthesis of isoquinolines is reported from internal alkenyl boronic esters, easily prepared from the corresponding 1,2-bis(boronates), via a sequential copper-catalyzed azidation / aza-Wittig condensation. This synthetic method has been used to synthesize quinisocaine, a topical anesthetic used for the treatment of pain and pruritus, and further extended to thieno[2,3-c]pyridines by using 2-thiophenecarboxaldehyde as coupling partner in the first step.

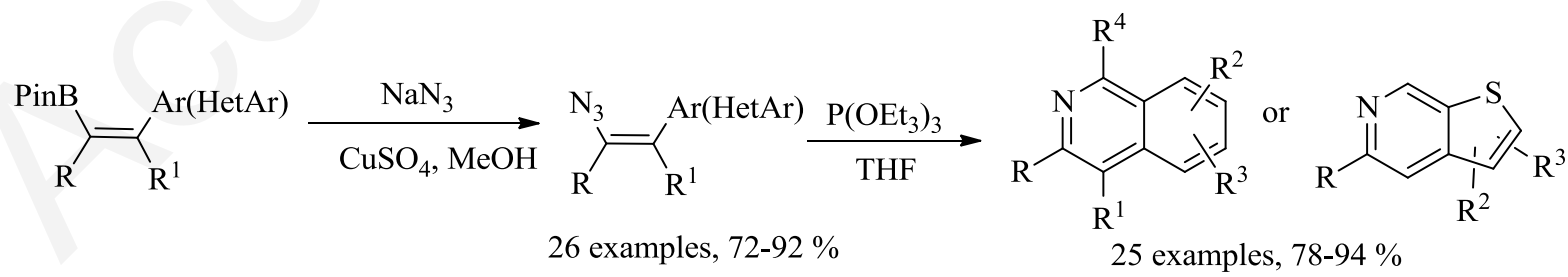


The isoquinoline core, and the related heterocycle-fused pyridine motifs, can be found in many natural products. ${ }^{1}$ These structural frameworks also constitute the common substructures of a large variety of biologically active substances and organic materials. ${ }^{2,3}$ Due to such diverse properties, rapid and efficient routes to access isoquinolines are still attracting considerable attention from organic chemists over the past few years and a number of different synthetic strategies were devised for these nitrogen heterocycles. If the BischlerNapieralski ${ }^{4}$ and Pomerantz-Fritsch ${ }^{5}$ reactions are classically used, alternative protocols, often possessing milder reaction conditions, wider substrate scope, and better functional compatibilities, are now available. ${ }^{6}$ During the past decade, transition-metal-catalyzed annulation reactions have proven to be valuable key process for the synthesis of isoquinolines. ${ }^{7}$ With respect to processes that do not use metal catalysts, intramolecular azaWittig reaction, ${ }^{8}$ which occurs under mild and neutral conditions, has been also exploited to access isoquinolines and isoquinolones. ${ }^{9}$ To the best of our knowledge, this approach was hitherto restricted to ester-substituted substrates resulting from the condensation reaction of an aldehyde with ethyl or methyl azidoacetate.

Organoboranes have recently emerged as valuable precursors of azido compounds via their copper(II)-catalyzed reaction with sodium or trimethylsilyl azide ${ }^{10}$ that greatly increases the range of available vinyl azides. In this context, and on the basis of our previous works on alkenyl 1,2-bis(boronic esters), ${ }^{11}$ herein we report a practical access to isoquinolines from these easily available starting material, based on a three steps sequence: regioselective Suzuki-Miyaura coupling with a 2-formyl aryl bromide / azidation / aza-Wittig condensation (Scheme 1).

Scheme 1. Synthesis of isoquinolines 4 from alkenyl 1,2-bis(boronic esters) 1

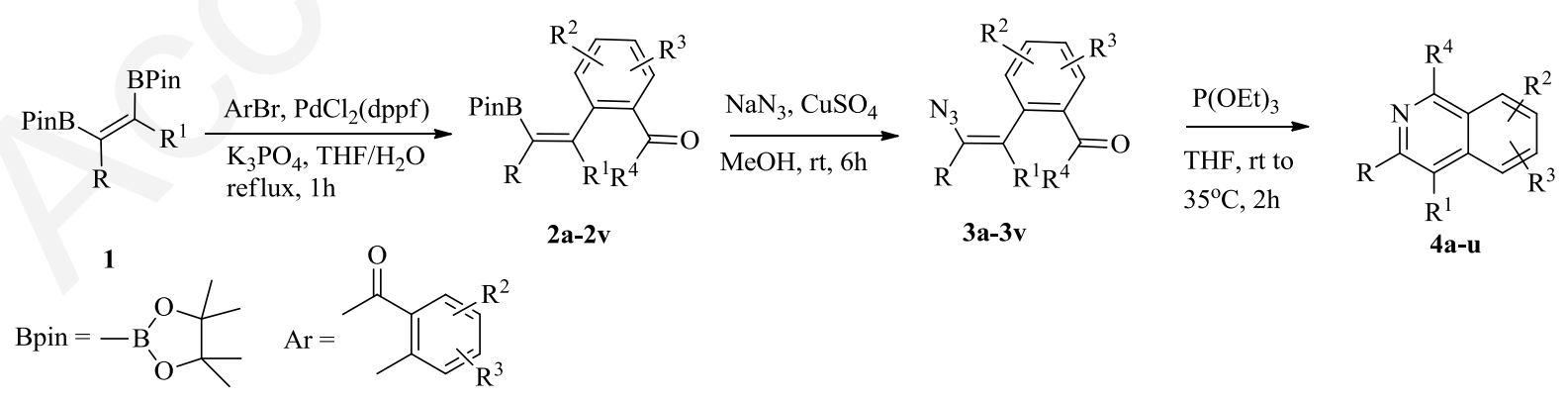


(E)-Alkenyl 1,2-bis(boronates) 1a-e were first prepared in good yields from the corresponding 1-alkynes and bis(pinacolato)diboron in the presence of tetrakis(triphenylphosphine)platinum as catalyst, according to reported procedures. ${ }^{12}$ These compounds were then regioselectively converted to the internal boronic esters via SuzukiMiyaura cross-couplings with 2-formyl aromatic halides in the presence of $\mathrm{Pd}(\mathrm{dppf}) \mathrm{Cl}_{2}$ and $\mathrm{K}_{3} \mathrm{PO}_{4}$ in $\mathrm{THF} / \mathrm{H}_{2} \mathrm{O}$ at reflux as previously reported. ${ }^{11}$ Yields are good to moderate with the formation of a single $(E)$-stereoisomer 2, as ascertained by NMR spectroscopy (Scheme 2).

Replacement of the aldehyde group by an ester, ketone or amide motif has no significant influence on the course of the coupling reaction.

Scheme 2. Suzuki-Miyaura cross-couplings with 1,2-bis(boronates) $\mathbf{1}^{\text {a,b }}$

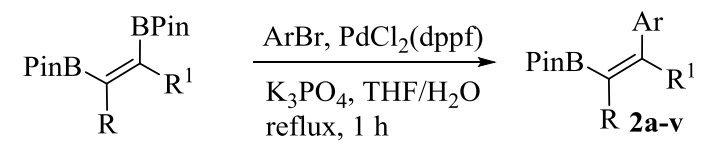
1a $\quad \mathrm{R}=n-\mathrm{Bu}, \mathrm{R}^{1}=\mathrm{H}$
1d $\mathrm{R}=\mathrm{C}_{3} \mathrm{H}_{6} \mathrm{OTBS}, \mathrm{R}^{1}=\mathrm{H}$
1b $\mathrm{R}=\mathrm{CH}_{2} \mathrm{Ph}, \mathrm{R}^{1}=\mathrm{H} \quad$ le $\mathrm{R}=\mathrm{R}^{1}=\mathrm{C}_{3} \mathrm{H}_{7}$
1c $\mathrm{R}=\mathrm{CH}_{2}-\mathrm{cHex}, \mathrm{R}^{1}=\mathrm{H}$

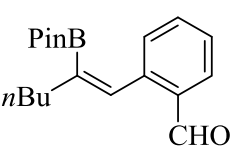

2a $(75 \%)$<smiles>CC/C([10BH]c1ccccc1)=C\c1ccc(OC)cc1C=O</smiles>

2b $(58 \%)$<smiles></smiles>

2c (56\%)<smiles>CCCCC([Pb])=Cc1c(C=O)ccc2ccccc12</smiles>

2d(51\%)<smiles>[CH]C/C([PH2+])=C\c1cc(OC)c(OC)cc1C=O</smiles>

2e $(52 \%)$<smiles>COc1cc(/C=C(/[Pb])CC(C)C)c(C=O)cc1O[SbH3]</smiles>

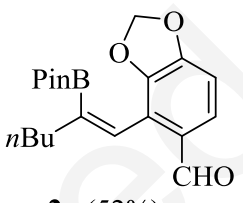<smiles>O=C[C]/C(=C/c1ccccc1C=O)Pc1ccccc1</smiles>

2h $(62 \%)$<smiles>C=[Ge]C/C(=C/c1ccc(OC)cc1C=O)[Pb]c1ccccc1</smiles>

$2 \mathbf{i}(52 \%)$<smiles>C=C/C=C([PH4+])\C=C/c1c(C=O)ccc2ccccc12</smiles><smiles>O=CC/C([PH])=C/c1c(C=O)ccc2c1OCO2</smiles><smiles>[CH]=CC/C(=C/c1cc(OC)c(O[SbH3])cc1C=O)Pc1ccccc1</smiles><smiles>COc1cc(C=O)c(/C=C(/C=O)c2ccccc2)cc1OC</smiles>

2o $(64 \%)$ 2k (57\%)<smiles>CC(COS(C)(=O)=O)C(C)Cc1ccccc1C=O</smiles>

2p $(58 \%)$ 2l (62\%)<smiles>[B]Oc1cc(C=O)c(NC(C)C(CO[Sb](C)(=O)=O)c2ccccc2)cc1OC</smiles>

$2 q(56 \%)$<smiles>O=Cc1ccccc1/C=C(/[Pb])Cc1ccccc1</smiles>

2m (52\%) $\mathrm{CHO}$<smiles>COc1ccc(/C=C(\Cc2ccccc2)c2ccccc2)c(C=O)c1</smiles>
2n $(58 \%)$<smiles>CCC(=Cc1ccc(F)cc1C=O)PC(C)C</smiles>

2r $(32 \%)$<smiles>CCCCC(=C(CC)CCC)c1ccccc1C=O</smiles><smiles>CCC/C(=C\c1ccccc1C(=O)OC)c1ccccc1</smiles>

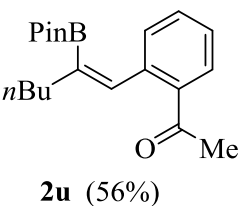<smiles>CCCCC(=Cc1ccccc1C(N)=O)Pc1ccccc1</smiles>

${ }^{a}$ General conditions: 1 ( $\left.0.5 \mathrm{mmol}\right)$, [1, 1'-bis (diphenyl phosphino)ferrocene] dichloropalladium(II) ( $\left.0.01 \mathrm{mmol}\right)$, potassium phosphate tribasic monohydrate $(1.5 \mathrm{mmol})$ and arylbromide $(0.5 \mathrm{mmol})$, THF $(5 \mathrm{~mL}) /$ water $(0.1 \mathrm{~mL})$, reflux, $1 \mathrm{~h} .{ }^{\mathrm{b}}$ Yields of isolated products. 
We then studied the copper-catalyzed transformation of compounds 2 to the corresponding azides in methanol that took place at room temperature after $6 \mathrm{~h}$, to give the azide in good yields ( 72 to $92 \%$ ). The reaction was compatible with the presence of various functionalities, including carbonyl groups, alkyl and silyl ethers, fluorine, ester and amide (Scheme 3).

Scheme 3. Copper-catalyzed conversion of boronates $\mathbf{2}$ into azides $\mathbf{3}^{\mathrm{a}}$,
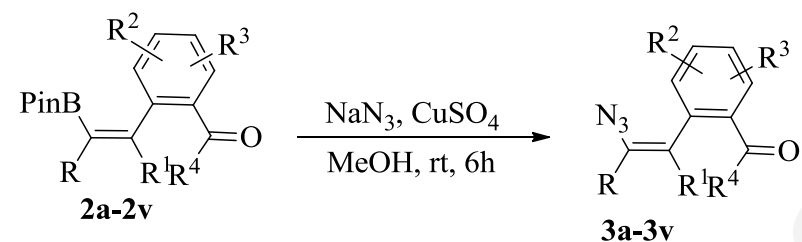

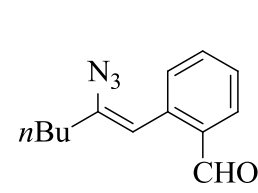

3a $(91 \%)$<smiles>COc1ccc(/C=C(\N)[15NH2])c(C=O)c1</smiles>

$\mathbf{3 b}(75 \%)$<smiles></smiles>

3c $(78 \%)$<smiles>C[18CH2]/C(N)=C/c1c(C=O)ccc2ccccc12</smiles>

3d $(76 \%)$<smiles>COc1cc(C=O)c(/C=C(/[15CH2])N)cc1OC</smiles>

3e $(92 \%)$<smiles>COc1cc(/C=C(/N)C[15NH2])c(C=O)cc1O[SbH3]</smiles>

3f $(85 \%)$<smiles>C[18NH]/C(N)=C/c1c(C=O)ccc2c1OCO2</smiles>

3g (75\%)<smiles>C=CC/C(N)=C/c1ccccc1C=O</smiles>

3h $(85 \%)$<smiles>COc1ccc(/C=C(\N)CC=O)c(C=O)c1</smiles>

3i $(79 \%)$<smiles>CCCC(N)=Cc1c(C=O)ccc2ccccc12</smiles>

3j (81\%)<smiles>N/C(=C\c1c(C=O)ccc2c1OCO2)CC=O</smiles>

3k (84\%)<smiles>C=CC/C(N)=C/c1cc(OC)c(O[SbH3])cc1C=O</smiles><smiles>N/C(=C\c1ccccc1C=O)Cc1ccccc1</smiles>

3m (78\%)<smiles>COc1ccc(C=C(N)Cc2ccccc2)c(C=O)c1</smiles>

3n $(72 \%)$<smiles>COc1cc(/C=C(\N)Cc2ccccc2)c(C=O)cc1OCCOCC(N)/C=C(\N)c1ccccc1C=O</smiles><smiles>COc1cc(/C=C(\N)[18OH])c(C=O)cc1O[13CH3]</smiles><smiles>CCCCC(C)=Cc1ccc(F)cc1C=O</smiles>

3r $(86 \%)$<smiles>CCCCCCC(=C(N)CCCC)c1ccccc1C=O</smiles>

3s $(84 \%)$<smiles>CCCC/C(N)=C\c1ccccc1C(=O)OC</smiles>

$3 t(79 \%)$<smiles>CCCCC(N)=Cc1ccccc1C(C)=O</smiles>

3u $(84 \%)$<smiles>CCCCC(C)=Cc1ccccc1C(N)=O</smiles>
3v $(86 \%)$

${ }^{\text {a }}$ General conditions: $2(0.5 \mathrm{mmol})$, sodium azide $(0.6 \mathrm{mmol})$ and copper sulfate $(0.3 \mathrm{mmol}), \mathrm{MeOH}(2 \mathrm{~mL}), \mathrm{rt}, 6 \mathrm{~h}$.

${ }^{\mathrm{b}}$ Yields of isolated products. 
We further investigated the substrate scope of the intramolecular aza-Wittig reaction of azidoaldehydes $\mathbf{3 a - 3 v}$ in the presence of triethylphosphite. The desired quinolines $\mathbf{4 a}-\mathbf{4} \mathbf{u}$ were isolated in yields ranging from $82 \%$ to $93 \%$ after 2 hours at $35{ }^{\circ} \mathrm{C}$ in THF (Scheme 4). This cyclization was not restricted to aldehyde derivatives and good yields were also observed with a ketone and an ester group. While, it failed with the amide $\mathbf{3 v}$.

Scheme 4. Synthesis of isoquinolines 4 from azides 3 . $^{\mathrm{a}, \mathrm{b}}$
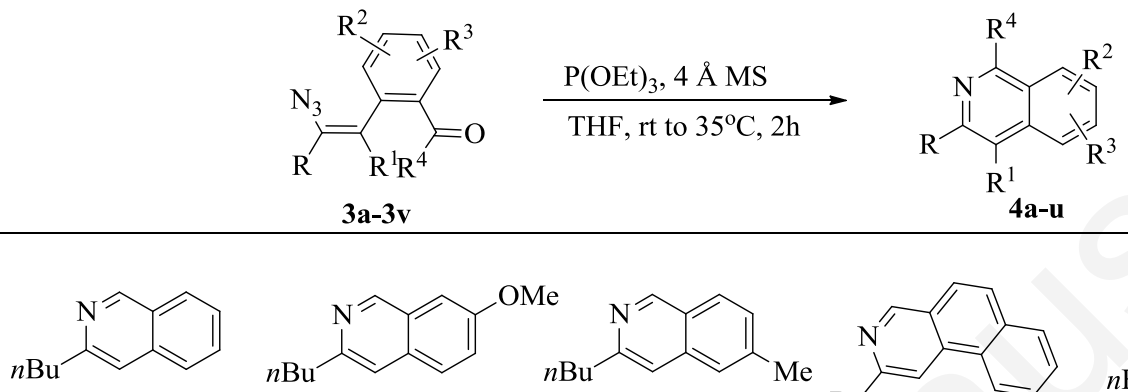

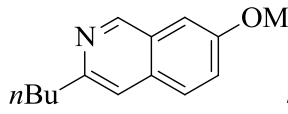

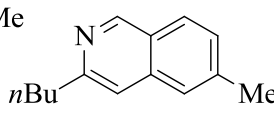

4a $(93 \%)$

4b $(85 \%)$ $4 \mathbf{c}(85 \%)$

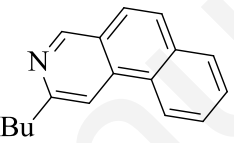

4d $(83 \%)$<smiles>CCCCCc1cc2cc(OC)c(OC)cc2cn1</smiles>

4e $(84 \%)$<smiles>CCCCc1cc2cc(OC)c(O[13CH3])cc2cn1</smiles>

$\mathbf{4 f}(90 \%)$<smiles>CC=CCc1cc2ccc(OC)cc2cn1</smiles>

4i $(89 \%)$<smiles>C=CCc1cc2c(ccc3ccccc32)cn1</smiles>

$4 \mathbf{j}(92 \%)$

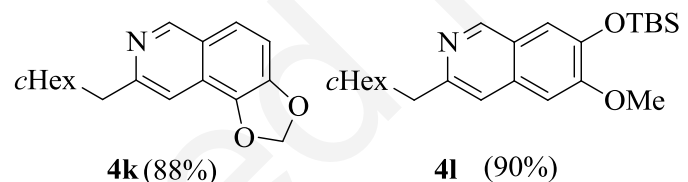<smiles>c1ccc(Cc2cc3ccccc3cn2)cc1</smiles><smiles>COc1ccc2cc(Cc3ccccc3)ncc2c1</smiles><smiles>COc1cc2cnc(Cc3ccccc3)cc2cc1OC</smiles><smiles>[SeH2]C1CC[AsH2]1</smiles><smiles>Cc1cc2ccccc2cn1</smiles>

$40 \quad(86 \%)$

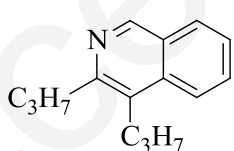

4s $(91 \%)$

$4 \mathbf{p}(85 \%)$<smiles>CCCCc1cc2ccccc2c(OC)n1</smiles>

4t $(87 \%)$<smiles>COC(=O)Nc1cc2cc(OC)c(O[Mg])cc2cn1</smiles>

$3 \mathbf{4 q}(92 \%)$<smiles>CCCCc1cc2ccccc2c(C)n1</smiles>

$4 u(88 \%)$<smiles>CCCCc1cc2ccc(F)cc2cn1</smiles>

4r $(91 \%)$

${ }^{\text {a }}$ General conditions: 3 (0.5 mmol) THF ( $\left.3 \mathrm{~mL}\right), 4$ Å molecular sieves and triethylphosphite $(0.5 \mathrm{mmol})$, THF $(4 \mathrm{~mL})$, rt, $30 \mathrm{~min}$; triethoxyphosphite $(1 \mathrm{mmol}), 35^{\circ} \mathrm{C}, 1.5 \mathrm{~h} .{ }^{\mathrm{b}}$ Yields of isolated products.

Like isoquinolines, thieno[2,3-c]pyridines, which belong to the related fused pyridine family, are an important class of biologically active molecules. ${ }^{13,14}$ Our above described approach was transposed to this class of heterocycles, to provide the starting Suzuki partner as a thiophene derivative. We thus succeeded to synthesize compounds $\mathbf{6 a - 6 \mathbf { d }}$ according to a 
three steps sequence in good overall yields from 3-bromothiophene-2-carboxaldehyde and 3bromobenzothiophene-2-carboxaldehyde selected as model heteroaromatic halides (Table 1).

Table 1. Synthesis of thieno[2,3-c]pyridines 8 from bisboronates $1{ }^{\mathrm{a}}$

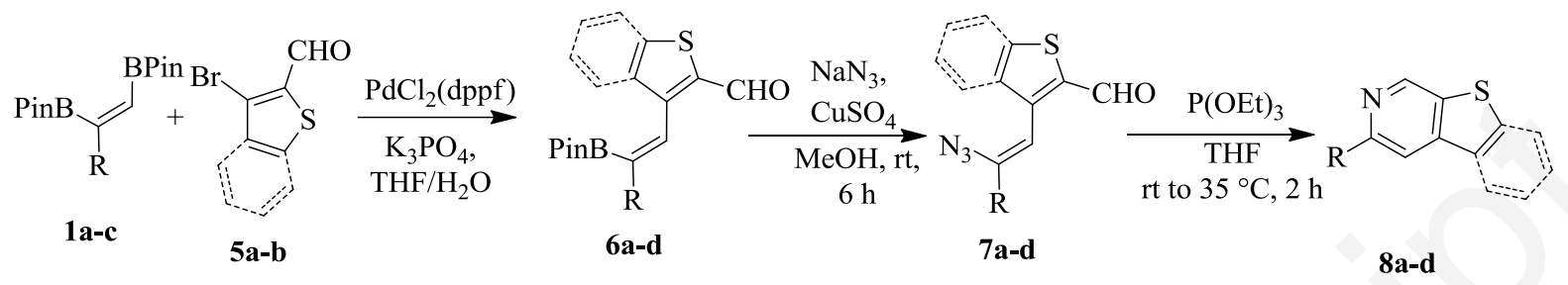

\begin{tabular}{|c|c|c|c|c|c|}
\hline \multirow{2}{*}{ Entry } & \multirow[b]{2}{*}{$\mathrm{R}$} & \multirow{2}{*}{$\begin{array}{l}\text { Heteroaromatic } \\
\text { halide }\end{array}$} & \multicolumn{3}{|c|}{ Yield(\%) } \\
\hline & & & 6 & 7 & 8 \\
\hline $\mathbf{a}$ & $n$-Bu & & 59 & 88 & 86 \\
\hline b & $\mathrm{CH}_{2} c \mathrm{Hex}$ & " & 64 & 92 & 94 \\
\hline c & $\mathrm{CH}_{2} \mathrm{Ph}$ & " & 58 & 87 & 88 \\
\hline d & $n$-Bu & & 52 & 75 & 78 \\
\hline
\end{tabular}

Finally, the utility of this sequence was demonstrated by the synthesis of quinisocaine, (dimethisoquin), a topical anesthetic used for the treatment of pain and pruritus. ${ }^{15}$ The boronate 10 was first synthesized from $1 \mathbf{a}$ and 2-(dimethylamino) ethyl 2-bromobenzoate 9 in a $62 \%$ yield. In presence of sodium azide and copper sulfate, it was readily converted to the corresponding azide 11, which after an intramolecular aza-Wittig condensation, gave quinisocaine 12 in $81 \%$ yield (Scheme 5).

Scheme 5. Synthesis of Quinisocaine.

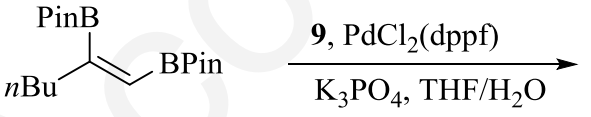

1a<smiles>CCCC(=Cc1ccccc1C(=O)OCCN(C)C)CC</smiles>

$10(62 \%)$<smiles>N[Mg]NC(=O)CCCC(=O)O</smiles>

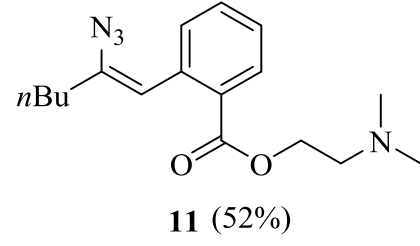

Quinisocaine 
In conclusion, an efficient and widely applicable synthesis of isoquinolines from easily accessible or commercially available reactants was developed. The key features of this method are a regioselective Suzuki coupling at the terminal C-B bond of a (E)-1-alkene-1,2diboronic ester, the copper-catalyzed azidation and intramolecular Aza-Wittig reactions. This approach has been successfully extended to thieno[2,3-c]pyridines and exemplified by the synthesis of quinisocaine, a topical anesthetic.

\section{EXPERIMENTAL SECTION}

General Information and Materials. All commercially available chemicals were used without further purification. Tetrahydrofuran (THF) and diethylether were used as received. Analytical thin layer chromatography was performed on Silica Gel 60 F254 plates. The compounds were characterized by ${ }^{1} \mathrm{H},{ }^{13} \mathrm{C}$ NMR and ${ }^{11} \mathrm{~B}$ NMR. Spectra were recorded in $\mathrm{CDCl}_{3}$ (internal standard: $7.26 \mathrm{ppm}$ for ${ }^{1} \mathrm{H} ; 77.00 \mathrm{ppm}$ for ${ }^{13} \mathrm{C}$ ). ${ }^{11} \mathrm{~B}$ NMR chemical shifts are related to external $\mathrm{BF}_{3} \cdot \mathrm{OEt}_{2}(0.0 \mathrm{ppm})$. High-resolution mass spectra (HMRS) were recorded on a micro-TOF-Q II mass analyzer or Q-TOF 2 using positive ion electrospray. Compounds 1 were synthesized according to literature procedures. ${ }^{12}$

\section{General Procedure for the Suzuki-Miyaura Cross-Couplings with 1, 2-Bis(boronates)}

(1a-1e). Synthesis of compounds $\mathbf{2 a - 2 v}$ and $\mathbf{6 a - 6 \boldsymbol { d }}$. A solution of bispinacolate ester 1 (0.89 $\mathrm{mmol})$ in THF $(8.9 \mathrm{~mL})$ and water $(0.18 \mathrm{~mL})$ was degassed under argon atmosphere before the addition of [1, 1'-bis (diphenyl phosphino)ferrocene] dichloropalladium(II) (13.5 mg, $0.018 \mathrm{mmol})$, potassium phosphate tribasic monohydrate $(616 \mathrm{mg}, 2.66 \mathrm{mmol})$ and arylbromide $(0.89 \mathrm{mmol})$. The reaction mixture was heated at reflux for $1 \mathrm{~h}$, cooled to room temperature, diluted with water and extracted with $\mathrm{Et}_{2} \mathrm{O}(2 \times 25 \mathrm{~mL})$. The combined organic extracts were dried $\left(\mathrm{MgSO}_{4}\right)$ and evaporated under vacuum. The residue was purified by column chromatography (230-400 mesh Silica gel, EtOAc in Hexane) to give the corresponding Suzuki products 2 or $\mathbf{6}$.

The characterisation of compounds $\mathbf{2 a}, \mathbf{2 b}, \mathbf{2 d}, \mathbf{2 e}, \mathbf{2 l}, \mathbf{2 n}$ and $\mathbf{2 o}$ was already reported in our previous paper. ${ }^{11}$ 
(E)-4-Methyl-2-(2-(4,4,5,5-tetramethyl-1,3,2-dioxaborolan-2-yl)hex-1-

enyl)benzaldehyde (2c). $164 \mathrm{mg}(56 \%)$. Colorless oil, $\mathrm{R}_{f}=0.10$ (EtOAc/Hexane 10:90). ${ }^{1} \mathrm{H}$ $\operatorname{NMR}\left(500 \mathrm{MHz}, \mathrm{CDCl}_{3}\right): \delta 10.22(\mathrm{~s}, 1 \mathrm{H}), 7.75(\mathrm{~d}, J=7.9 \mathrm{~Hz}, 1 \mathrm{H}), 7.30(\mathrm{~s}, 1 \mathrm{H}), 7.16(\mathrm{~d}, J=$ $7.9 \mathrm{~Hz}, 1 \mathrm{H}), 7.11(\mathrm{~s}, 1 \mathrm{H}), 2.37-2.34(\mathrm{~m}, 5 \mathrm{H}), 1.58-1.45(\mathrm{~m}, 2 \mathrm{H}), 1.39(\mathrm{td}, J=14.6,7.2 \mathrm{~Hz}$, 2H), 1.12 (s, 12H), $0.94(\mathrm{t}, J=7.3 \mathrm{~Hz}, 3 \mathrm{H}) .{ }^{13} \mathrm{C} \mathrm{NMR}\left(101 \mathrm{MHz}, \mathrm{CDCl}_{3}\right): \delta$ 192.0, 143.7, $142.9,136.5,131.5,130.8,128.5,128.1,83.4,37.2,31.8,24.5,22.4,21.7,14.0$ (the carbon $\alpha$ to boron was not found). HRMS (ESI+): $m / z(\mathrm{M}+\mathrm{H})^{+}$calculated for $\mathrm{C}_{20} \mathrm{H}_{30} \mathrm{O}_{3} \mathrm{~B} 329.2288$, found 329.2289 .

(E)-5-(tert-Butyldimethylsilyloxy)-4-methoxy-2-(2-(4,4,5,5-tetramethyl-1,3,2-

dioxaborolan-2-yl)hex-1-enyl)benzaldehyde (2f). $245 \mathrm{mg}$ (58\%). Colorless oil, $\mathrm{R}_{f}=0.10$ (EtOAc/Hexane 10:90). ${ }^{1} \mathrm{H}$ NMR (400 MHz, $\mathrm{CDCl}_{3}$ ): $\delta 10.07$ (s, 1H), 7.36 (s, 1H), 7.17 (s, $1 \mathrm{H}), 6.74(\mathrm{~s}, 1 \mathrm{H}), 3.87(\mathrm{~s}, 3 \mathrm{H}), 2.41-2.28(\mathrm{~m}, 2 \mathrm{H}), 1.50$ (ddd, $J=14.6,9.4,4.0 \mathrm{~Hz}, 2 \mathrm{H}), 1.44-$ $1.35(\mathrm{~m}, 2 \mathrm{H}), 1.09(\mathrm{~s}, 12 \mathrm{H}), 0.99(\mathrm{~s}, 9 \mathrm{H}), 0.94(\mathrm{t}, J=7.2 \mathrm{~Hz}, 3 \mathrm{H}), 0.15(\mathrm{~s}, 6 \mathrm{H}) .{ }^{13} \mathrm{C} \mathrm{NMR}$ (126 MHz, $\left.\mathrm{CDCl}_{3}\right): \delta 190.9,155.2,144.3,141.8$ (br), 139.2, 135.9, 127.6, 118.9, 112.8, 83.4, 55.5, 37.1, 31.9, 25.7, 24.6, 22.4, 18.5, 14.0, -4. HRMS (ESI+): $m / z(\mathrm{M}+\mathrm{H})^{+}$calculated for $\mathrm{C}_{26} \mathrm{H}_{44} \mathrm{O}_{5} \mathrm{BSi}$ 475.3051, found 475.3041.

(E)-4-(2-(4,4,5,5-Tetramethyl-1,3,2-dioxaborolan-2-yl)hex-1-enyl)benzo[d][1,3]dioxole5-carbaldehyde (2g). $166 \mathrm{mg}$ (52\%). Colourless oil, $\mathrm{R}_{f}=0.50\left(\right.$ EtOAc/Hexane 20:80). ${ }^{1} \mathrm{H}$ NMR (500 MHz, $\left.\mathrm{CDCl}_{3}\right): \delta 10.26(\mathrm{~s}, 1 \mathrm{H}), 7.19$ (s, 1H), 6.88 (d, $\left.J=7.9 \mathrm{~Hz}, 1 \mathrm{H}\right), 6.74$ (dd, $J$ $=8.0,0.6 \mathrm{~Hz}, 1 \mathrm{H}), 6.12(\mathrm{~s}, 2 \mathrm{H}), 2.38-2.28(\mathrm{~m}, 2 \mathrm{H}), 1.52-1.45(\mathrm{~m}, 2 \mathrm{H}), 1.42-1.33(\mathrm{~m}, 2 \mathrm{H})$, $1.15(\mathrm{~s}, 12 \mathrm{H}), 0.93(\mathrm{t}, J=7.3 \mathrm{~Hz}, 3 \mathrm{H}) .{ }^{13} \mathrm{C} \mathrm{NMR}\left(101 \mathrm{MHz}, \mathrm{CDCl}_{3}\right): \delta 189.5,148.5,147.7$, 136.3, 135.6, 123.0, 117.5, 112.2, 102.5, 83.4, 37.0, 31.8, 24.7, 24.6, 22.4, 14.0 (the carbon $\alpha$ to boron was not found). HRMS (ESI+): $m / z(M+H)^{+}$calculated for $\mathrm{C}_{20} \mathrm{H}_{28} \mathrm{O}_{5} \mathrm{~B}$ 359.2030, found.359.2030.

(E)-2-(3-Cyclohexyl-2-(4,4,5,5-tetramethyl-1,3,2-dioxaborolan-2-yl)prop-1-

enyl)benzaldehyde (2h). $175 \mathrm{mg}(62 \%)$. Colourless oil, $\mathrm{R}_{f}=0.10$ (EtOAc/Hexane 10:90). ${ }^{1} \mathrm{H}$ NMR $\left(400 \mathrm{MHz}, \mathrm{CDCl}_{3}\right): \delta 10.29(\mathrm{~d}, J=0.5 \mathrm{~Hz}, 1 \mathrm{H}), 7.86(\mathrm{dd}, J=7.7,1.2 \mathrm{~Hz}, 1 \mathrm{H}), 7.46$ $(\mathrm{td}, J=7.5,1.4 \mathrm{~Hz}, 1 \mathrm{H}), 7.35(\mathrm{t}, J=7.5 \mathrm{~Hz}, 1 \mathrm{H}), 7.29(\mathrm{~d}, J=7.5 \mathrm{~Hz}, 1 \mathrm{H}), 7.26(\mathrm{~s}, 1 \mathrm{H}), 2.27$ $(\mathrm{dd}, J=7.1,1.0 \mathrm{~Hz}, 2 \mathrm{H}), 1.82-1.65(\mathrm{~m}, 5 \mathrm{H}), 1.46$ (dtd, $J=10.9,7.2,3.6 \mathrm{~Hz}, 1 \mathrm{H}), 1.28-1.18$ $(\mathrm{m}, 3 \mathrm{H}), 1.09(\mathrm{~s}, 12 \mathrm{H}), 0.96-.93(\mathrm{~m}, 2 \mathrm{H}) .{ }^{13} \mathrm{C}$ NMR $\left(126 \mathrm{MHz}, \mathrm{CDCl}_{3}\right): \delta$ 192.4, 143.0, $137.4,133.7,133.0,130.4,127.9,127.2,83.4,45.6,38.0,33.3,26.5,26.4,24.5$ (the carbon $\alpha$ to boron was not found). HRMS (ESI+): $m / z(\mathrm{M}+\mathrm{Na})^{+}$calculated for $\mathrm{C}_{22} \mathrm{H}_{31} \mathrm{O}_{3} \mathrm{BNa}$ 377.2264, found 377.2267 . 
(E)-2-(3-Cyclohexyl-2-(4,4,5,5-tetramethyl-1,3,2-dioxaborolan-2-yl)prop-1-enyl)-5methoxybenzaldehyde (2i). $159 \mathrm{mg}(52 \%)$. Colourless oil, $\mathrm{R}_{f}=0.30$ (EtOAc/Hexane 10:90). ${ }^{1} \mathrm{H}$ NMR $\left(500 \mathrm{MHz}, \mathrm{CDCl}_{3}\right): \delta 10.26(\mathrm{~s}, 1 \mathrm{H}), 7.36(\mathrm{~d}, J=2.8 \mathrm{~Hz}, 1 \mathrm{H}), 7.22(\mathrm{~d}, J=8.5 \mathrm{~Hz}$, $1 \mathrm{H}), 7.18(\mathrm{~s}, 1 \mathrm{H}), 7.04(\mathrm{dd}, J=8.5,2.8 \mathrm{~Hz}, 1 \mathrm{H}), 3.85$ (s, 3H), $2.25(\mathrm{dd}, J=7.1,1.0 \mathrm{~Hz}, 2 \mathrm{H})$, 1.78-1.70 (m, 5H), 1.53-1.38 (m, 1H), 1.29-1.17 (m, 3H), $1.11(\mathrm{~s}, 12 \mathrm{H}), 1.01-0.87(\mathrm{~m}, 2 \mathrm{H})$. ${ }^{13} \mathrm{C}$ NMR (126 MHz, $\left.\mathrm{CDCl}_{3}\right): \delta$ 192.0, 158.8, 140.1, 139.8 (br), 136.8, 136.3, 134.5, 131.7, 120.8, 109.7, 83.4, 55.5, 45.7, 38.0, 33.3, 26.5, 26.4, 24.5. HRMS (ESI+): $m / z(\mathrm{M}+\mathrm{Na})^{+}$ calculated for $\mathrm{C}_{23} \mathrm{H}_{33} \mathrm{BO}_{4} \mathrm{Na} 407.2369$, found 407.2343.

(E)-1-(3-Cyclohexyl-2-(4,4,5,5-tetramethyl-1,3,2-dioxaborolan-2-yl)prop-1-enyl)-2naphthaldehyde (2j). $174 \mathrm{mg}(54 \%)$. Colorless oil, $\mathrm{R}_{f}=0.20$ (EtOAc/Hexane 10:90). ${ }^{1} \mathrm{H}$ NMR (400 MHz, $\left.\mathrm{CDCl}_{3}\right): \delta 10.45(\mathrm{~d}, J=0.8 \mathrm{~Hz}, 1 \mathrm{H}), 8.12(\mathrm{dd}, J=8.3,0.7 \mathrm{~Hz}, 1 \mathrm{H}), 7.96$ (d, $J=8.6 \mathrm{~Hz}, 1 \mathrm{H}), 7.87-7.75(\mathrm{~m}, 2 \mathrm{H}), 7.56(\mathrm{ddd}, J=8.3,6.9,1.3 \mathrm{~Hz}, 2 \mathrm{H}), 7.34(\mathrm{~s}, 1 \mathrm{H}), 2.46$ (dd, $J=22.9,6.7 \mathrm{~Hz}, 2 \mathrm{H}), 1.91-1.65(\mathrm{~m}, 5 \mathrm{H}), 1.58-1.50(\mathrm{~m}, 1 \mathrm{H}), 1.33-1.17$ (m, 3H), 1.05 (d, $J=10.6 \mathrm{~Hz}, 2 \mathrm{H}), 0.86(\mathrm{~s}, 6 \mathrm{H}), 0.78(\mathrm{~s}, 6 \mathrm{H}) .{ }^{13} \mathrm{C} \mathrm{NMR}\left(75 \mathrm{MHz}, \mathrm{CDCl}_{3}\right): \delta 193.0,144.3$, 135.7, 134.5, 132.5, 131.5, 128.4, 128.1, 127.3, 126.9, 126.4, 121.9, 83.2, 45.5, 38.1, 33.5, 26.5, 26.4, 24.2 (the carbon $\alpha$ to boron was not found). HRMS (ESI+): $m / z(\mathrm{M}+\mathrm{Na})^{+}$ calculated for $\mathrm{C}_{26} \mathrm{H}_{33} \mathrm{O}_{3} \mathrm{BNa}$ 427.2420, found 427.2424.

(E)-4-(3-Cyclohexyl-2-(4,4,5,5-tetramethyl-1,3,2-dioxaborolan-2-yl)prop-1enyl)benzo[d][1,3]dioxole-5-carbaldehyde (2k). $181 \mathrm{mg}$ (57\%). Yellow solid (m.p = 81-85 $\left.{ }^{\circ} \mathrm{C}\right), \mathrm{R}_{f}=0.50\left(\right.$ EtOAc/Hexane 20:80). ${ }^{1} \mathrm{H}$ NMR (400 MHz, $\mathrm{CDCl}_{3}$ ): $\delta 10.26(\mathrm{~s}, 1 \mathrm{H}), 7.14$ (s, $1 \mathrm{H}), 6.88(\mathrm{~d}, J=7.9 \mathrm{~Hz}, 1 \mathrm{H}), 6.74(\mathrm{dd}, J=8.0,0.8 \mathrm{~Hz}, 1 \mathrm{H}), 6.12(\mathrm{~s}, 2 \mathrm{H}), 2.22(\mathrm{dd}, J=7.1$, $0.9 \mathrm{~Hz}, 2 \mathrm{H}), 1.81-1.65(\mathrm{~m}, 5 \mathrm{H}), 1.44(\mathrm{ddd}, J=11.0,7.4,3.6 \mathrm{~Hz}, 1 \mathrm{H}), 1.25-1.15(\mathrm{~m}, 3 \mathrm{H}), 1.14$ (s, 12H), 0.99-0.85 (m, 2H). ${ }^{13} \mathrm{C} \mathrm{NMR}\left(101 \mathrm{MHz}, \mathrm{CDCl}_{3}\right): \delta 189.6,148.3,147.7,137.3$, $135.6,123.1,117.5,112.2,102.5,83.4,45.5,37.9,33.3,26.5,26.4,24.6$ (the carbon $\alpha$ to boron was not found). HRMS (ESI+): $m / z(\mathrm{M}+\mathrm{Na})^{+}$calculated for $\mathrm{C}_{23} \mathrm{H}_{31} \mathrm{O}_{5} \mathrm{BNa} 421.2162$, found 421.2162 .

(E)-2-(3-Phenyl-2-(4,4,5,5-tetramethyl-1,3,2-dioxaborolan-2-yl)prop-1-

enyl)benzaldehyde (2m). $151 \mathrm{mg}(52 \%)$. Colourless oil, $\mathrm{R}_{f}=0.10$ (EtOAc/Hexane 10:90). ${ }^{1} \mathrm{H}$ NMR (400 MHz, $\left.\mathrm{CDCl}_{3}\right): \delta 10.27(\mathrm{~s}, 1 \mathrm{H}), 7.85(\mathrm{dd}, J=7.7,1.4 \mathrm{~Hz}, 1 \mathrm{H}), 7.51-7.43(\mathrm{~m}, 1 \mathrm{H})$, 7.40-7.34 (m, 2H), 7.33-7.28 (m, 5H), 7.23-7.14 (m, 1H), $3.70(\mathrm{~d}, J=1.4 \mathrm{~Hz}, 2 \mathrm{H}), 0.96$ (s, 12H). $\left.{ }^{13} \mathrm{C} \mathrm{NMR} \mathrm{(101} \mathrm{MHz,} \mathrm{CDCl}_{3}\right): \delta 192.2,142.4,139.9,137.9,133.7,133.0,130.42$, 129.2, 128.6, 128.3, 127.4, 126.1, 83.5, 43.4, 24.4 (the carbon $\alpha$ to boron was not found). HRMS (ESI+): $m / z(M+H)^{+}$calculated for $\mathrm{C}_{22} \mathrm{H}_{26} \mathrm{O}_{3} \mathrm{~B} 349.1975$, found 349.1970. 
(E)-2-(5-(tert-Butyldimethylsilyloxy)-2-(4,4,5,5-tetramethyl-1,3,2-dioxaborolan-2-

yl)pent-1-enyl)benzaldehyde (2p). $154 \mathrm{mg}$ (58\%). Colorless oil, $\mathrm{R}_{f}=0.10$ (EtOAc/Hexane 10:90). ${ }^{1} \mathrm{H}$ NMR (400 MHz, $\left.\mathrm{CDCl}_{3}\right): \delta 10.27$ (s, 1H), 7.86 (dd, $\left.J=7.7,1.0 \mathrm{~Hz}, 1 \mathrm{H}\right), 7.47$ (td, $J=7.5,1.2 \mathrm{~Hz}, 1 \mathrm{H}), 7.40-7.27(\mathrm{~m}, 3 \mathrm{H}), 3.68(\mathrm{t}, J=6.5 \mathrm{~Hz}, 2 \mathrm{H}), 2.48-2.32(\mathrm{~m}, 2 \mathrm{H}), 1.82-$ $1.64(\mathrm{~m}, 2 \mathrm{H}), 1.09$ (s, 12H), 0.99-0.85 (m, 12H), 0.07 (s, 6H). $\left.{ }^{13} \mathrm{C} \mathrm{NMR} \mathrm{(101} \mathrm{MHz,} \mathrm{CDCl}\right)$ : $\delta 192.2,142.9,136.8,133.7,133.0,130.3,128.0,127.2,83.5,62.7,33.8,32.8,26.0,24.5$, $18.3,-5.1$ (the carbon $\alpha$ to boron was not found). HRMS (ESI+): $m / z\left(\mathrm{M}+\mathrm{NH}_{4}\right)^{+}$calculated for $\mathrm{C}_{24} \mathrm{H}_{43} \mathrm{NO}_{4} \mathrm{BSi} 448.3054$, found 448.3055 .

(E)-5-(tert-Butyldimethylsilyloxy)-2-(5-(tert-butyldimethylsilyloxy)-2-(4,4,5,5tetramethyl-1,3,2-dioxaborolan-2-yl)pent-1-enyl)-4-methoxybenzaldehyde (2q). $219 \mathrm{mg}$ (56\%). Colorless oil, $\mathrm{R}_{f}=0.30\left(\right.$ EtOAc/Hexane 10:90). ${ }^{1} \mathrm{H}$ NMR $\left(400 \mathrm{MHz}, \mathrm{CDCl}_{3}\right): \delta 10.06$ (s, 1H), $7.36(\mathrm{~s}, 1 \mathrm{H}), 7.20(\mathrm{~s}, 1 \mathrm{H}), 6.74(\mathrm{~s}, 1 \mathrm{H}), 3.87(\mathrm{~s}, 3 \mathrm{H}), 3.68(\mathrm{t}, J=6.5 \mathrm{~Hz}, 2 \mathrm{H}), 2.39$ (dd, $J=8.0,6.6 \mathrm{~Hz}, 2 \mathrm{H}), 1.79-1.70(\mathrm{~m}, 2 \mathrm{H}), 1.08(\mathrm{~s}, 12 \mathrm{H}), 0.99(\mathrm{~s}, 9 \mathrm{H}), 0.91(\mathrm{~s}, 9 \mathrm{H}), 0.15$ (s, 6H), 0.07 (s, 6H). ${ }^{13} \mathrm{C}$ NMR (101 MHz, $\left.\mathrm{CDCl}_{3}\right): \delta 190.8,155.2,144.3,139.1,136.4,127.6$, $118.9,112.8,83.4,62.8,55.5,33.6,32.9,26.0,25.7,24.6,18.5,18.3,-4.6,-5.1$ (the carbon $\alpha$ to boron was not found). HRMS (ESI+): $m / z(\mathrm{M}+\mathrm{Na})^{+}$calculated for $\mathrm{C}_{31} \mathrm{H}_{55} \mathrm{O}_{6} \mathrm{BNaSi}_{2}$ 613.3528, found 613.3538.

(E)-5-Fluoro-2-(2-(4,4,5,5-tetramethyl-1,3,2-dioxaborolan-2-yl)hex-1enyl)benzaldehyde (2r). $95 \mathrm{mg}$ (32\%). Colourless oil, $\mathrm{R}_{f}=0.10$ (EtOAc/Hexane 5:95). ${ }^{1} \mathrm{H}$ NMR (400 MHz, $\left.\mathrm{CDCl}_{3}\right): \delta 10.22(\mathrm{~d}, J=3.0 \mathrm{~Hz}, 1 \mathrm{H}), 7.54(\mathrm{dd}, J=9.0,2.8 \mathrm{~Hz}, 1 \mathrm{H}), 7.29-$ $7.24(\mathrm{~m}, 1 \mathrm{H}), 7.17(\mathrm{dt}, J=8.3,2.8 \mathrm{~Hz}, 2 \mathrm{H}), 2.36(\mathrm{td}, J=7.7,1.3 \mathrm{~Hz}, 2 \mathrm{H}), 1.55-1.44(\mathrm{~m}, 2 \mathrm{H})$, 1.41-1.34 (m, 2H), $1.09(\mathrm{~s}, 12 \mathrm{H}), 0.94(\mathrm{t}, J=7.2 \mathrm{~Hz}, 3 \mathrm{H}) .{ }^{13} \mathrm{C} \mathrm{NMR}\left(126 \mathrm{MHz}, \mathrm{CDCl}_{3}\right): \delta$ 191.0, 161.9 (d, $J=248 \mathrm{~Hz}), 139.3(\mathrm{~d}, J=2.6 \mathrm{~Hz}), 135.4(\mathrm{~d}, J=6 \mathrm{~Hz}), 135.2,132.3(\mathrm{~d}, J=7$ Hz), $120.1(\mathrm{~d}, J=22 \mathrm{~Hz}), 113.4(\mathrm{~d}, J=22 \mathrm{~Hz}), 83.5,37.1,31.8,24.5,22.4,14.0$ (the carbon $\alpha$ to boron was not found). HRMS (ESI+): $m / z(\mathrm{M}+\mathrm{Na})^{+}$calculated for $\mathrm{C}_{19} \mathrm{H}_{26} \mathrm{O}_{3} \mathrm{BFNa}$ 355.1857 , found 355.1856 .

(E)-2-(5-(4,4,5,5-Tetramethyl-1,3,2-dioxaborolan-2-yl)oct-4-en-4-yl)benzaldehyde (2s). 155 $\mathrm{mg}(55 \%)$. Yellow solid (m.p $\left.=52-55{ }^{\circ} \mathrm{C}\right), \mathrm{R}_{f}=0.10\left(\right.$ EtOAc/Hexane 5:95). ${ }^{1} \mathrm{H}$ NMR (400 $\left.\mathrm{MHz}_{\mathrm{CDCl}}\right): \delta 10.15(\mathrm{~d}, J=0.6 \mathrm{~Hz}, 1 \mathrm{H}), 7.89(\mathrm{dd}, J=7.8,1.1 \mathrm{~Hz}, 1 \mathrm{H}), 7.47(\mathrm{td}, J=7.5$, $1.4 \mathrm{~Hz}, 1 \mathrm{H}), 7.33$ (t, $J=7.5 \mathrm{~Hz}, 1 \mathrm{H}), 7.18(\mathrm{dd}, J=7.6,0.7 \mathrm{~Hz}, 1 \mathrm{H}), 2.52-2.26(\mathrm{~m}, 4 \mathrm{H}), 1.52-$ $1.44(\mathrm{~m}, 2 \mathrm{H}), 1.38-1.24(\mathrm{~m}, 2 \mathrm{H}), 0.98(\mathrm{t}, J=7.3 \mathrm{~Hz}, 3 \mathrm{H}), 0.93-0.84(\mathrm{~m}, 15 \mathrm{H}) .{ }^{13} \mathrm{C}$ NMR $(101$ $\left.\mathrm{MHz}, \mathrm{CDCl}_{3}\right): \delta 192.9,149.2,146.4,134.3,132.9,130.2,126.7,126.3,82.9,37.3,33.0,24.3$, 
23.2, 20.7, 14.6, 14.3 (the carbon $\alpha$ to boron was not found). HRMS (ESI+): $m / z(M+H)^{+}$ calculated for $\mathrm{C}_{21} \mathrm{H}_{32} \mathrm{O}_{3} \mathrm{~B} 343.2444$, found 343.2444.

(E)-Methyl 2-(2-(4,4,5,5-tetramethyl-1,3,2-dioxaborolan-2-yl)hex-1-enyl)benzoate (2t). $160 \mathrm{mg}$ (52\%). Colorless oil, $\mathrm{R}_{f}=0.20$ (EtOAc/Hexane 10:90). ${ }^{1} \mathrm{H}$ NMR (400 MHz, $\left.\mathrm{CDCl}_{3}\right)$ : $\delta 7.92(\mathrm{dd}, J=7.8,1.1 \mathrm{~Hz}, 1 \mathrm{H}), 7.41$ (s, 1H), 7.39-7.34 (m, 1H), 7.31 (ddd, $J=6.4,3.5,1.2$ $\mathrm{Hz}, 1 \mathrm{H}), 7.28-7.25(\mathrm{~m}, 1 \mathrm{H}), 3.86(\mathrm{~s}, 3 \mathrm{H}), 2.33$ (ddd, $J=7.7,1.2,1.2 \mathrm{~Hz}, 2 \mathrm{H}), 1.56-1.44$ (m, $2 \mathrm{H}), 1.42-1.36(\mathrm{~m}, 2 \mathrm{H}), 1.14(\mathrm{~s}, 12 \mathrm{H}), 0.93$ (t, $J=7.2 \mathrm{~Hz}, 3 \mathrm{H}) .{ }^{13} \mathrm{C} \mathrm{NMR}\left(126 \mathrm{MHz}, \mathrm{CDCl}_{3}\right)$ : $\delta 167.7,141.4,140.4,131.3,130.8,130.2,128.4,126.7,83.2,51.8,37.1,31.9,24.6,22.4$, 14.0 (the carbon $\alpha$ to boron was not found). HRMS (ESI+): $m / z(\mathrm{M}+\mathrm{Na})^{+}$calculated for $\mathrm{C}_{20} \mathrm{H}_{29} \mathrm{O}_{4} \mathrm{BNa}$ 367.2057, found 367.2068.

(E)-1-(2-(2-(4,4,5,5-Tetramethyl-1,3,2-dioxaborolan-2-yl)hex-1-enyl)phenyl)ethanone (2u). $164 \mathrm{mg}$ (56\%). Colorless oil, $\mathrm{R}_{f}=0.30$ (EtOAc/Hexane 10:90). ${ }^{1} \mathrm{H}$ NMR (500 MHz, $\left.\mathrm{CDCl}_{3}\right): \delta 7.65(\mathrm{dd}, J=8.0,1.3 \mathrm{~Hz}, 1 \mathrm{H}), 7.37-7.32(\mathrm{~m}, 1 \mathrm{H}), 7.31-7.26(\mathrm{~m}, 2 \mathrm{H}), 7.23(\mathrm{~s}, 1 \mathrm{H})$, $2.55(\mathrm{~s}, 3 \mathrm{H}), 2.37-2.25(\mathrm{~m}, 2 \mathrm{H}), 1.53-1.45(\mathrm{~m}, 2 \mathrm{H}), 1.43-1.35(\mathrm{~m}, 2 \mathrm{H}), 1.13(\mathrm{~s}, 12 \mathrm{H}), 0.93(\mathrm{t}$, $J=7.3 \mathrm{~Hz}, 3 \mathrm{H}) .{ }^{13} \mathrm{C} \mathrm{NMR}\left(126 \mathrm{MHz}, \mathrm{CDCl}_{3}\right):{ }^{13} \mathrm{C} \mathrm{NMR}\left(126 \mathrm{MHz}, \mathrm{CDCl}_{3}\right): \delta 201.5,140.4$, 139.6, 137.6, 130.9, 130.9, 128.6, 126.9, 83.2, 37.1, 31.9, 30.1, 24.6, 22.5, 14.1 (the carbon $\alpha$ to boron was not found). HRMS (ESI+): $m / z(\mathrm{M}+\mathrm{Na})^{+}$calculated for $\mathrm{C}_{20} \mathrm{H}_{29} \mathrm{O}_{3} \mathrm{BNa} 351.2107$, found 351.2112 .

(E)-2-(2-(4,4,5,5-Tetramethyl-1,3,2-dioxaborolan-2-yl)hex-1-enyl)benzamide (2v). 183 $\mathrm{mg}(62 \%)$. Colorless oil, $\mathrm{R}_{f}=0.40\left(\mathrm{MeOH} \mathrm{CHCl}_{3} 10: 90\right) .{ }^{1} \mathrm{H} \mathrm{NMR}\left(500 \mathrm{MHz}, \mathrm{CDCl}_{3}\right): \delta$ $7.67(\mathrm{~d}, J=6.9 \mathrm{~Hz}, 1 \mathrm{H}), 7.31(\mathrm{t}, J=6.6 \mathrm{~Hz}, 2 \mathrm{H}), 7.20(\mathrm{~d}, J=6.8 \mathrm{~Hz}, 1 \mathrm{H}), 7.11(\mathrm{~s}, 1 \mathrm{H}), 6.45$ (br, 1H), 5.72 (br, 1H), 2.32 (t, $J=7.3 \mathrm{~Hz}, 2 \mathrm{H}), 1.50-1.44(\mathrm{~m}, 2 \mathrm{H}), 1.39-1.33(\mathrm{~m}, 2 \mathrm{H}), 1.10$ $(\mathrm{s}, 12 \mathrm{H}), 0.93(\mathrm{t}, J=7.2 \mathrm{~Hz}, 3 \mathrm{H}) .{ }^{13} \mathrm{C} \mathrm{NMR}\left(101 \mathrm{MHz}, \mathrm{CDCl}_{3}\right): \delta 171.2,138.9,137.7,133.8$, $130.1,130.0,128.1,127.3,83.6,36.6,31.6,29.7,24.5,22.4,13.9$ (the carbon $\alpha$ to boron was not found). HRMS (ESI+): $m / z(\mathrm{M}+\mathrm{Na})^{+}$calculated for $\mathrm{C}_{19} \mathrm{H}_{28} \mathrm{O}_{3} \mathrm{NBNa} 352.2060$, found 352.2086 .

\section{(E)-3-(2-(4,4,5,5-Tetramethyl-1,3,2-dioxaborolan-2-yl)hex-1-enyl)thiophene-2-}

carbaldehyde (6a). $168 \mathrm{mg}(59 \%)$. Colorless oil, $\mathrm{R}_{f}=0.20$ (EtOAc/Hexane 10:90). ${ }^{1} \mathrm{H}$ NMR (500 MHz, $\left.\mathrm{CDCl}_{3}\right): \delta 9.95(\mathrm{~d}, J=1.2 \mathrm{~Hz}, 1 \mathrm{H}), 7.56(\mathrm{dd}, J=5.0,1.1 \mathrm{~Hz}, 1 \mathrm{H}), 7.19-7.11(\mathrm{~m}$, 1H), $7.06(\mathrm{~s}, 1 \mathrm{H}), 2.36(\mathrm{td}, J=7.7,1.3 \mathrm{~Hz}, 2 \mathrm{H}), 1.52-1.46(\mathrm{~m}, 2 \mathrm{H}), 1.41-1.35(\mathrm{~m}, 2 \mathrm{H}), 1.18$ (s, 12H), $0.93(\mathrm{t}, J=7.3 \mathrm{~Hz}, 3 \mathrm{H}) .{ }^{13} \mathrm{C} \mathrm{NMR}\left(101 \mathrm{MHz}, \mathrm{CDCl}_{3}\right): \delta 183.4,149.0,138.8,132.9$, 130.6, 130.1, 83.8, 37.6, 31.7, 24.7, 22.4, 13.9 (the carbon $\alpha$ to boron was not found). HRMS (ESI+): $m / z(\mathrm{M}+\mathrm{Na})^{+}$calculated for $\mathrm{C}_{17} \mathrm{H}_{25} \mathrm{O}_{3} \mathrm{BNaS} 343.1515$, found 343.1513 . 
(E)-3-(3-Cyclohexyl-2-(4,4,5,5-tetramethyl-1,3,2-dioxaborolan-2-yl)prop-1-

enyl)thiophene-2-carbaldehyde (6b). $184 \mathrm{mg}$ (64\%). Yellow solid (m.p = 55-58 ${ }^{\circ} \mathrm{C}$ ), $\mathrm{R}_{f}=$ 0.20 (EtOAc/Hexane 10:90). ${ }^{1} \mathrm{H}$ NMR $\left(500 \mathrm{MHz}, \mathrm{CDCl}_{3}\right): \delta 9.96(\mathrm{~d}, J=1.0 \mathrm{~Hz}, 1 \mathrm{H}), 7.56$ $(\mathrm{dd}, J=5.0,0.6 \mathrm{~Hz}, 1 \mathrm{H}), 7.15(\mathrm{~d}, J=5.0 \mathrm{~Hz}, 1 \mathrm{H}), 7.01(\mathrm{~s}, 1 \mathrm{H}), 2.25(\mathrm{~d}, J=7.1 \mathrm{~Hz}, 2 \mathrm{H})$, 1.80-1.57 (m, 5H), 1.52-1.38 (m, 1H), 1.31-1.11 (m, 3H), 1.08 (s, 12H), 0.99-0.84 (m, 2H). ${ }^{13} \mathrm{C}$ NMR $\left(126 \mathrm{MHz}, \mathrm{CDCl}_{3}\right): \delta 183.5,148.9,138.8,132.9,131.6,130.2,83.7,46.0,37.9$, 33.3, 26.5, 26.3, 24.6 (the carbon $\alpha$ to boron was not found). HRMS (ESI+): $m / z(\mathrm{M}+\mathrm{Na})^{+}$ calculated for $\mathrm{C}_{20} \mathrm{H}_{29} \mathrm{O}_{3} \mathrm{BNaS} 383.1828$, found 383.1831 .

(E)-3-(3-Phenyl-2-(4,4,5,5-tetramethyl-1,3,2-dioxaborolan-2-yl)prop-1-enyl)thiophene-2carbaldehyde (6c). $167 \mathrm{mg}(58 \%)$. White solid ( $\left.\mathrm{m} . \mathrm{p}=95-98{ }^{\circ} \mathrm{C}\right), \mathrm{R}_{f}=0.30$ (EtOAc/Hexane 10:90). ${ }^{1} \mathrm{H}$ NMR (400 MHz, $\left.\mathrm{CDCl}_{3}\right): \delta 9.95$ (d, $\left.J=1.1 \mathrm{~Hz}, 1 \mathrm{H}\right), 7.57(\mathrm{dd}, J=5.0,0.9 \mathrm{~Hz}$, 1H), 7.32-7.21 (m, 5H), 7.19 (d, $J=5.3 \mathrm{~Hz}, 1 \mathrm{H}), 7.11(\mathrm{~s}, 1 \mathrm{H}), 3.69$ (d, $J=1.1 \mathrm{~Hz}, 2 \mathrm{H}), 1.04$ (s, 12H). ${ }^{13} \mathrm{C}$ NMR $\left(126 \mathrm{MHz}, \mathrm{CDCl}_{3}\right): \delta 183.2,148.3,139.4,139.1,133.0,131.9,130.1$, $129.2,128.4,126.3,83.8,43.8,24.5$ (the carbon $\alpha$ to boron was not found). HRMS (ESI+): $m / z\left(\mathrm{M}^{+}\right)$calculated for $\mathrm{C}_{20} \mathrm{H}_{23} \mathrm{O}_{3} \mathrm{BS} 354.1461$, found 354.1460.

(E)-3-(2-(4,4,5,5-Tetramethyl-1,3,2-dioxaborolan-2-yl)hex-1-enyl)benzo[b]thiophene-2carbaldehyde (6d). $172 \mathrm{mg}$ (52\%). Colourless oil, $\mathrm{R}_{f}=0.10$ (EtOAc/Hexane 10:90). ${ }^{1} \mathrm{H}$ NMR (500 MHz, $\left.\mathrm{CDCl}_{3}\right): \delta 10.08(\mathrm{~s}, 1 \mathrm{H}), 7.89-7.78(\mathrm{~m}, 2 \mathrm{H}), 7.52-7.45(\mathrm{~m}, 1 \mathrm{H}), 7.40$ (ddd, $J=8.1$, 7.1, 1.1 Hz, 1H), $7.06(\mathrm{~s}, 1 \mathrm{H}), 2.47$ (td, $J=7.6,1.3 \mathrm{~Hz}, 2 \mathrm{H}), 1.62-1.52(\mathrm{~m}, 2 \mathrm{H}), 1.44-1.43(\mathrm{~m}$, 2H), 1.00 (s, 12H), 0.98 (t, $\left.J=7.4 \mathrm{~Hz}, 3 \mathrm{H}) .13 \mathrm{C} \mathrm{NMR} \mathrm{(101} \mathrm{MHz,} \mathrm{CDCl}_{3}\right): \delta 185.9,145.5$, $141.3,139.7,139.1,129.9,127.9,124.9,124.7,123.0,83.6,37.2,31.8,24.4,22.4,14.0$ (the carbon $\alpha$ to boron was not found). HRMS (ESI+): $\mathrm{m} / z(\mathrm{M}+\mathrm{H})^{+}$calculated for $\mathrm{C}_{21} \mathrm{H}_{28} \mathrm{O}_{3} \mathrm{BS}$ 371.1852 , found 371.1851 .

General Procedure for the Synthesis of vinyl Azides from Boronates 2 or 6a. Synthesis of compounds $3 \boldsymbol{a}-3 \boldsymbol{u}$ and $7 \boldsymbol{a}-\mathbf{7 d}$. To a stirred solution of boronic ester 2 or $\mathbf{6}(0.477 \mathrm{mmol})$ in $\mathrm{MeOH}(2 \mathrm{~mL})$, sodium azide $(0.572 \mathrm{mmol})$ and copper sulfate $(0.298 \mathrm{mmol})$ were added at room temperature and stirred for $6 \mathrm{~h}$. The reaction mixture was diluted with water and extracted with $\mathrm{CH}_{2} \mathrm{Cl}_{2}(2 \times 5 \mathrm{~mL})$. The combined organic extracts were dried $\left(\mathrm{MgSO}_{4}\right)$ and evaporated under vacuum. The resulting residue was purified by column chromatography (230-400 mesh Silica gel, EtOAc in Hexane) to give vinyl azide 3 or 7 in 69-92\% yield.

(Z)-2-(2-Azidohex-1-enyl)benzaldehyde (3a). $99 \mathrm{mg}$ (91\%). Colorless oil, $\mathrm{R}_{f}=0.10$ (EtOAc/Hexane 10:90). ${ }^{1} \mathrm{H}$ NMR (500 MHz, $\left.\mathrm{CDCl}_{3}\right): \delta 10.15(\mathrm{~s}, 1 \mathrm{H}), 7.83(\mathrm{dd}, J=7.7,1.3$ 
$\mathrm{Hz}, 1 \mathrm{H}), 7.63(\mathrm{~d}, J=7.8 \mathrm{~Hz}, 1 \mathrm{H}), 7.54(\mathrm{td}, J=7.6,1.4 \mathrm{~Hz}, 1 \mathrm{H}), 7.37(\mathrm{td}, J=7.7,0.9 \mathrm{~Hz}$, $1 \mathrm{H}), 6.30(\mathrm{~s}, 1 \mathrm{H}), 2.49$ (dd, $J=11.5,3.8 \mathrm{~Hz}, 2 \mathrm{H}), 1.70-1.63(\mathrm{~m}, 2 \mathrm{H}), 1.51-148(\mathrm{~m}, 2 \mathrm{H}), 1.00$ $(\mathrm{t}, J=7.3 \mathrm{~Hz}, 3 \mathrm{H}) .{ }^{13} \mathrm{C} \mathrm{NMR}\left(101 \mathrm{MHz}, \mathrm{CDCl}_{3}\right): \delta 192.4,139.3,137.7,133.3,133.0,130.6$, 130.4, 127.1, 111.1, 33.4, 30.0, 22.1, 13.8. IR (neat): 3056, 2957, 2930, 2865, 2110, 1695, 1630, 1591. HRMS (ESI+): $m / z\left(\mathrm{M}-\mathrm{N}_{2}+\mathrm{H}\right)^{+}$calculated for $\mathrm{C}_{13} \mathrm{H}_{16} \mathrm{ON} 202.1232$, found 202.1231 .

(Z)-2-(2-Azidohex-1-enyl)-5-methoxybenzaldehyde (3b). $85 \mathrm{mg}$ (75\%). Colorless oil, $\mathrm{R}_{f}=$ 0.10 (EtOAc/Hexane 10:90). ${ }^{1} \mathrm{H}$ NMR (500 MHz, $\left.\mathrm{CDCl}_{3}\right): \delta 10.13$ (s, 1H), 7.53-7.46 (m, $1 \mathrm{H}), 7.35(\mathrm{~d}, J=2.9 \mathrm{~Hz}, 1 \mathrm{H}), 7.14-7.08(\mathrm{~m}, 1 \mathrm{H}), 6.15(\mathrm{~s}, 1 \mathrm{H}), 3.86$ (s, 3H), 2.50-2.45 (m, $2 \mathrm{H}), 1.67-1.64(\mathrm{~m}, 2 \mathrm{H}), 1.53-1.44(\mathrm{~m}, 2 \mathrm{H}), 0.99(\mathrm{t}, J=7.3 \mathrm{~Hz}, 3 \mathrm{H}) .{ }^{13} \mathrm{C}$ NMR $(126 \mathrm{MHz}$, $\left.\mathrm{CDCl}_{3}\right): \delta 191.9,158.6,138.6,134.0,131.7,130.9,120.7,112.4,110.4,55.5,33.3,30.0$,

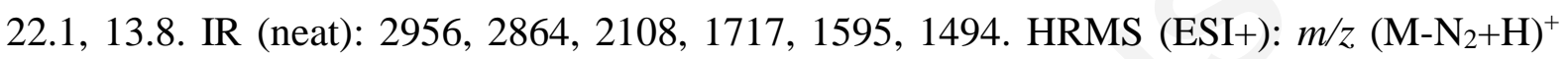
calculated for $\mathrm{C}_{14} \mathrm{H}_{18} \mathrm{NO}_{2} 232.1338$, found 232.1345.

(Z)-2-(2-Azidohex-1-enyl)-5-methylbenzaldehyde (3c). $87 \mathrm{mg}$ (78\%). Colorless oil, $\mathrm{R}_{f}=$ 0.10 (EtOAc/Hexane 5:95). ${ }^{1} \mathrm{H}$ NMR (400 MHz, $\left.\mathrm{CDCl}_{3}\right): \delta 10.09$ (s, $\left.1 \mathrm{H}\right), 7.73(\mathrm{~d}, J=7.9 \mathrm{~Hz}$, 1H), $7.42(\mathrm{~s}, 1 \mathrm{H}), 7.18(\mathrm{~d}, J=7.9 \mathrm{~Hz}, 1 \mathrm{H}), 6.27$ (s, 1H), 2.53-2.45 (m, 2H), 2.42 (s, 3H), 1.72$1.62(\mathrm{~m}, 2 \mathrm{H}), 1.51-1.47(\mathrm{~m}, 2 \mathrm{H}), 1.00(\mathrm{t}, J=7.3 \mathrm{~Hz}, 3 \mathrm{H}) .{ }^{13} \mathrm{C} \mathrm{NMR}\left(126 \mathrm{MHz}, \mathrm{CDCl}_{3}\right): \delta$ 192.1, 144.2, 139.0, 137.6, 130.9, 130.90, 130.8, 128.0, 111.2, 33.4, 30.0, 22.1, 21.9, 13.8. IR

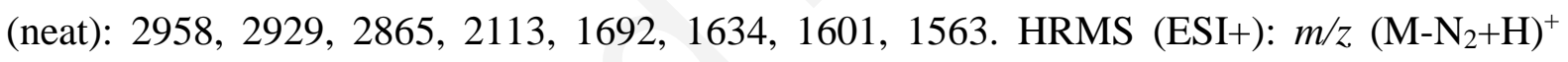
calculated for $\mathrm{C}_{14} \mathrm{H}_{18} \mathrm{ON} 216.1382$, found 216.1396.

(Z)-1-(2-Azidohex-1-enyl)naphthalene (3d). $87 \mathrm{mg}$ (76\%). Colorless oil, $\mathrm{R}_{f}=0.10$ (EtOAc/Hexane 5:95). ${ }^{1} \mathrm{H}$ NMR (300 MHz, $\left.\mathrm{CDCl}_{3}\right): \delta 10.31(\mathrm{~s}, 1 \mathrm{H}), 8.07$ (d, $J=8.2 \mathrm{~Hz}$, 1H), 7.99 (d, $J=8.6 \mathrm{~Hz}, 1 \mathrm{H}), 7.86(\mathrm{t}, J=9.3 \mathrm{~Hz}, 2 \mathrm{H}), 7.63(\mathrm{dd}, J=10.2,6.2 \mathrm{~Hz}, 1 \mathrm{H}), 7.59$ $(\mathrm{dd}, J=10.0,6.3 \mathrm{~Hz}, 1 \mathrm{H}), 6.22(\mathrm{~s}, 1 \mathrm{H}), 2.69-2.58$ (m, 2H), 1.88-1.69 (m, 2H), 1.61-1.56 (m, 2H), $1.06(\mathrm{t}, J=7.3 \mathrm{~Hz}, 3 \mathrm{H}) .{ }^{13} \mathrm{C} \mathrm{NMR}\left(75 \mathrm{MHz}, \mathrm{CDCl}_{3}\right): \delta 192.6,142.5,139.0,136.0$, 131.6, 131.0, 128.7, 128.6, 128.1, 126.8, 126.0, 122.4, 33.1, 30.1, 22.3, 13.8. IR (neat) :

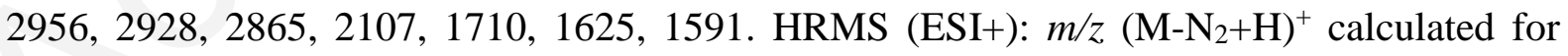
$\mathrm{C}_{17} \mathrm{H}_{18} \mathrm{NO} 252.1388$, found 252.1391.

(Z)-2-(2-Azidohex-1-enyl)-4,5-dimethoxybenzaldehyde (3e). 107 mg (92\%). Colorless oil, $\mathrm{R}_{f}=0.30\left(\right.$ EtOAc/Hexane 10:90). ${ }^{1} \mathrm{H}$ NMR (500 MHz, $\left.\mathrm{CDCl}_{3}\right): \delta 10.07$ (s, 1H), $7.36(\mathrm{~s}, 1 \mathrm{H})$, $7.06(\mathrm{~s}, 1 \mathrm{H}), 6.18(\mathrm{~s}, 1 \mathrm{H}), 3.97(\mathrm{~s}, 3 \mathrm{H}), 3.93(\mathrm{~s}, 3 \mathrm{H}), 2.54-2.43(\mathrm{~m}, 2 \mathrm{H}), 1.72-1.62(\mathrm{~m}, 2 \mathrm{H})$, 1.54-1.43 (m, 2H), $1.00(\mathrm{t}, J=7.3 \mathrm{~Hz}, 3 \mathrm{H}) .{ }^{13} \mathrm{C} \mathrm{NMR}\left(101 \mathrm{MHz}, \mathrm{CDCl}_{3}\right): \delta 190.3,153.3$, 148.2, 139.1, 133.2, 126.5, 112.0, 110.18, 110.1, 56.1, 56.0, 33.4, 30.0, 22.1, 13.8. IR (neat): 


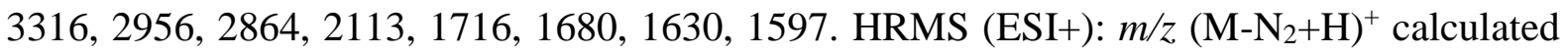
for $\mathrm{C}_{15} \mathrm{H}_{20} \mathrm{NO}_{3} 262.1443$, found 262.1446 .

(Z)-2-(2-Azidohex-1-enyl)-5-(tert-butyldimethylsilyloxy)-4-methoxybenzaldehyde (3f). $105 \mathrm{mg}(85 \%)$. Colorless oil, $\mathrm{R}_{f}=0.10$ (EtOAc/Hexane 10:90). ${ }^{1} \mathrm{H}$ NMR (400 MHz, $\left.\mathrm{CDCl}_{3}\right)$ : $\delta 9.99$ (s, 1H), 7.29 (s, 1H), $7.14(\mathrm{~s}, 1 \mathrm{H}), 6.29$ (s, 1H), 3.90 (s, 1H), 2.54-2.41 (m, 2H), 1.68$1.64(\mathrm{~m}, 2 \mathrm{H}), 1.51-1.47(\mathrm{~m}, 2 \mathrm{H}), 1.03-0.95(\mathrm{~m}, 12 \mathrm{H}), 0.19-0.14(\mathrm{~m}, 6 \mathrm{H}) .{ }^{13} \mathrm{C}$ NMR (101 $\left.\mathrm{MHz}, \mathrm{CDCl}_{3}\right): \delta 190.7,155.3,144.1,138.3,133.1,126.7,121.7,112.71,110.7,55.6,55.5$, 33.6, 30.1, 25.6, 22.1, 18.4, 13.8, -4.5. IR (neat):3303, 2956, 2931, 2858, 2112, 1682, 1601. HRMS (ESI+): $m / z\left(M-\mathrm{N}_{2}+\mathrm{H}\right)^{+}$calculated for $\mathrm{C}_{20} \mathrm{H}_{32} \mathrm{O}_{3} \mathrm{NSi} 362.2152$, found 362.2171.

(Z)-4-(2-Azidohex-1-enyl)benzo[d][1,3]dioxole-5-carbaldehyde (3g). 86 mg (75\%). Colorless oil, $\mathrm{R}_{f}=0.20$ (EtOAc/Hexane 10:90). ${ }^{1} \mathrm{H}$ NMR (500 MHz, $\left.\mathrm{CDCl}_{3}\right): \delta 10.19$ (s, 1H), $7.08(\mathrm{~d}, J=8.1 \mathrm{~Hz}, 1 \mathrm{H}), 6.96(\mathrm{~d}, J=8.1 \mathrm{~Hz}, 1 \mathrm{H}), 6.18(\mathrm{~s}, 1 \mathrm{H}), 6.11(\mathrm{~s}, 2 \mathrm{H}), 2.49-2.41(\mathrm{~m}$, 2H), 1.69-1.59 (m, 2H), 1.52-1.41 (m, 2H), $0.99(\mathrm{t}, J=7.3 \mathrm{~Hz}, 3 \mathrm{H}) .{ }^{13} \mathrm{C}$ NMR $(126 \mathrm{MHz}$, $\left.\mathrm{CDCl}_{3}\right): \delta 188.9,149.9,147.3,138.1,130.0,123.4,116.9,111.2,102.5,33.3,29.7,22.1$, 14.0, 13.8. HRMS (ESI+): $\mathrm{m} / z,\left(\mathrm{M}-\mathrm{N}_{2}+\mathrm{H}\right)^{+}$calculated for $\mathrm{C}_{14} \mathrm{H}_{16} \mathrm{NO}_{3}$ 246.1130, found 246.1132 .

(Z)-2-(2-Azido-3-cyclohexylprop-1-enyl)benzaldehyde (3h). $97 \mathrm{mg}$ (85\%). Colorless oil, $\mathrm{R}_{f}=0.10\left(\right.$ EtOAc/Hexane 10:90). ${ }^{1} \mathrm{H}$ NMR $\left(400 \mathrm{MHz}, \mathrm{CDCl}_{3}\right): \delta 10.16(\mathrm{~s}, 1 \mathrm{H}), 7.83$ (dd, $J=$ 7.7, $1.3 \mathrm{~Hz}, 1 \mathrm{H}), 7.64(\mathrm{~d}, J=7.8 \mathrm{~Hz}, 1 \mathrm{H}), 7.54(\mathrm{td}, J=7.6,1.4 \mathrm{~Hz}, 1 \mathrm{H}), 7.37(\mathrm{td}, J=7.7,1.1$ $\mathrm{Hz}, 1 \mathrm{H}), 6.25(\mathrm{~s}, 1 \mathrm{H}), 2.37(\mathrm{~d}, J=7.2 \mathrm{~Hz}, 2 \mathrm{H}), 1.89$ (d, $J=1.8 \mathrm{~Hz}, 1 \mathrm{H}), 1.87(\mathrm{~d}, J=1.8 \mathrm{~Hz}$, $1 \mathrm{H}), 1.82-1.73(\mathrm{~m}, 3 \mathrm{H}), 1.61-1.57(\mathrm{~m}, 1 \mathrm{H}), 1.34-1.18(\mathrm{~m}, 3 \mathrm{H}), 1.05-1.02(\mathrm{~m}, 2 \mathrm{H}) .{ }^{13} \mathrm{C}$ NMR $\left(101 \mathrm{MHz}, \mathrm{CDCl}_{3}\right): \delta 192.4,137.9,137.6,133.3,132.9,130.5,130.4,127.1,112.4,41.8$, 36.5, 33.0, 26.3, 26.1. IR (neat) : 2924, 2850, 2112, 1696, 1630, 1594. HRMS (ESI+): $\mathrm{m} / \mathrm{z}$ $\left(\mathrm{M}-\mathrm{N}_{2}+\mathrm{H}\right)^{+}$calculated for $\mathrm{C}_{16} \mathrm{H}_{20} \mathrm{ON} 242.1545$, found 242.1554 .

(Z)-2-(2-Azido-3-cyclohexylprop-1-enyl)-5-methoxybenzaldehyde (3i). $92 \mathrm{mg}$ (79\%). Colorless oil, $\mathrm{R}_{f}=0.10\left(\right.$ EtOAc/Hexane 10:90). ${ }^{1} \mathrm{H}$ NMR $\left(400 \mathrm{MHz}, \mathrm{CDCl}_{3}\right): \delta 10.14(\mathrm{~s}, 1 \mathrm{H})$, $7.51(\mathrm{~d}, J=8.6 \mathrm{~Hz}, 1 \mathrm{H}), 7.35(\mathrm{~d}, J=2.9 \mathrm{~Hz}, 1 \mathrm{H}), 7.18-7.02(\mathrm{~m}, 1 \mathrm{H}), 6.10(\mathrm{~s}, 1 \mathrm{H}), 3.86(\mathrm{~s}$, $3 \mathrm{H}), 2.35(\mathrm{~d}, J=7.2 \mathrm{~Hz}, 2 \mathrm{H}), 1.92-1.65(\mathrm{~m}, 5 \mathrm{H}), 1.60-1.58(\mathrm{~m}, 1 \mathrm{H}), 1.38-1.14(\mathrm{~m}, 3 \mathrm{H}), 1.04-$ $1.01(\mathrm{~m}, 2 \mathrm{H}) .{ }^{13} \mathrm{C}$ NMR $\left(126 \mathrm{MHz}, \mathrm{CDCl}_{3}\right): \delta 191.8,158.6,137.1,134.0,131.7,130.9,120.7$, 112.3, 111.8, 55.5, 41.6, 36.5, 33.0, 26.3, 26.1. IR (neat): 2924, 2849, 2113, 1690, 1635, 1599. HRMS (ESI+): $\mathrm{m} / z\left(\mathrm{M}-\mathrm{N}_{2}+\mathrm{H}\right)^{+}$calculated for $\mathrm{C}_{17} \mathrm{H}_{22} \mathrm{O}_{2} \mathrm{~N} 272.1651$, found 272.1662.

(Z)-1-(2-Azido-3-cyclohexylprop-1-enyl)-2-naphthaldehyde (3j). $96 \mathrm{mg}$ (81\%). Colorless oil, $\mathrm{R}_{f}=0.10$ (EtOAc/Hexane 5:95). ${ }^{1} \mathrm{H}$ NMR (400 MHz, $\left.\mathrm{CDCl}_{3}\right): \delta 10.32(\mathrm{~d}, J=0.8 \mathrm{~Hz}, 1 \mathrm{H})$, $8.08(\mathrm{dd}, J=8.3,0.7 \mathrm{~Hz}, 1 \mathrm{H}), 7.99(\mathrm{~d}, J=8.6 \mathrm{~Hz}, 1 \mathrm{H}), 7.89-7.81(\mathrm{~m}, 2 \mathrm{H}), 7.65-7.54(\mathrm{~m}$, 
2H), $6.19(\mathrm{~s}, 1 \mathrm{H}), 2.52(\mathrm{~d}, J=7.1 \mathrm{~Hz}, 2 \mathrm{H}), 2.03-1.92(\mathrm{~m}, 2 \mathrm{H}), 1.87-1.78(\mathrm{~m}, 2 \mathrm{H}), 1.78-1.66$ (m, 2H), 1.40-1.24 (m, 3H), 1.20-1.08 (m, 2H). ${ }^{13} \mathrm{C}$ NMR (126 MHz, $\left.\mathrm{CDCl}_{3}\right): \delta 192.6,141.1$, 139.0, 136.0, 131.7, 131.0, 128.7, 128.63, 128.2, 126.8, 125.9, 122.4, 109.2, 41.6, 36.4, 33.2, 26.2, 26.1. IR (neat) : 2924, 2850, 2114, 1684, 1642, 1591. HRMS (ESI+): m/z (M$\left.\mathrm{N}_{2}+\mathrm{H}\right)^{+}$calculated for $\mathrm{C}_{20} \mathrm{H}_{22} \mathrm{ON} 292.1701$, found 292.1712.

(Z)-4-(2-Azido-3-cyclohexylprop-1-enyl)benzo[d][1,3]dioxole-5-carbaldehyde (3k). 99 mg (84\%). Colorless oil, $\mathrm{R}_{f}=0.10\left(\right.$ EtOAc/Hexane 10:90). ${ }^{1} \mathrm{H}$ NMR $\left(500 \mathrm{MHz}, \mathrm{CDCl}_{3}\right): \delta$ 10.20 (s, 1H), 7.09 (d, $J=8.1 \mathrm{~Hz}, 1 \mathrm{H}), 6.96$ (d, $J=8.1 \mathrm{~Hz}, 1 \mathrm{H}), 6.13(\mathrm{~s}, 1 \mathrm{H}), 6.11(\mathrm{~s}, 2 \mathrm{H})$, $2.33(\mathrm{~d}, J=7.2 \mathrm{~Hz}, 2 \mathrm{H}), 1.86(\mathrm{~d}, J=13.2 \mathrm{~Hz}, 2 \mathrm{H}), 1.81-1.68(\mathrm{~m}, 3 \mathrm{H}), 1.63-1.58(\mathrm{~m}, 1 \mathrm{H})$, 1.35-1.26 (m, 3H), 1.09-0.96 (m, 2H). $\left.{ }^{13} \mathrm{C} \mathrm{NMR} \mathrm{(101} \mathrm{MHz,} \mathrm{CDCl}_{3}\right): \delta 188.9,149.8,147.3$, 136.7, 130.0, 123.5, 116.9, 112.7, 112.6, 102.5, 41.7, 36.5, 32.9, 26.3, 26.1. IR (neat): 2923, 2853, 2115, 1688, 1630. HRMS (ESI+): $m / z\left(M-\mathrm{N}_{2}+\mathrm{H}\right)^{+}$calculated for $\mathrm{C}_{17} \mathrm{H}_{20} \mathrm{O}_{3} \mathrm{~N}$ 286.1443, found 286.1454 .

\section{(Z)-2-(2-Azido-3-cyclohexylprop-1-enyl)-5-(tert-butyldimethylsilyloxy)-4-}

methoxybenzaldehyde (3l). $103 \mathrm{mg}$ (82\%). Colorless oil, $\mathrm{R}_{f}=0.10$ (EtOAc/Hexane 10:90). ${ }^{1} \mathrm{H}$ NMR (500 MHz, $\left.\mathrm{CDCl}_{3}\right) \delta 10.00$ (s, 1H), 7.29 (s, 1H), 7.15 (s, 1H), 6.25 (s, 1H), 3.90 (s, $3 \mathrm{H}), 2.35(\mathrm{~d}, J=7.1 \mathrm{~Hz}, 2 \mathrm{H}), 1.87(\mathrm{~d}, J=12.0, \mathrm{~Hz}, 2 \mathrm{H}), 1.77(\mathrm{~d}, J=12.9 \mathrm{~Hz}, 2 \mathrm{H}), 1.64-1.50$ (m, 2H), 1.37-1.21 (m, 3H), 1.11-0.90 (m, 11H), 0.17 (s, 6H). ${ }^{13} \mathrm{C}$ NMR (100 MHz, $\left.\mathrm{CDCl}_{3}\right)$ : $\delta 190.6,155.4,144.0,136.7,133.1,126.6,121.5,112.6,112.0,55.5,41.9,36.4,32.9,26.1$, 26.2, 25.6, 18.5, -4.6. IR (neat) : 2928, 2854, 2116, 1685, 1629, 1595. HRMS (ESI+): $\mathrm{m} / \mathrm{z}$ $\left(\mathrm{M}-\mathrm{N}_{2}+\mathrm{H}\right)^{+}$calculated for $\mathrm{C}_{23} \mathrm{H}_{36} \mathrm{O}_{3} \mathrm{NSi} 402.2465$, found 402.2482 .

(Z)-2-(2-Azido-3-phenylprop-1-enyl)benzaldehyde(3m). $88 \mathrm{mg}(78 \%)$. Colorless oil, $\mathrm{R}_{f}=$ 0.10 (EtOAc/Hexane 10:90). ${ }^{1} \mathrm{H}$ NMR (500 MHz, $\left.\mathrm{CDCl}_{3}\right) \delta 10.14(\mathrm{~s}, 1 \mathrm{H}), 7.83(\mathrm{~d}, J=7.5$ $\mathrm{Hz}, 1 \mathrm{H}), 7.68$ (d, $J=7.6 \mathrm{~Hz}, 1 \mathrm{H}), 7.55$ (t, $J=7.3 \mathrm{~Hz}, 1 \mathrm{H}), 7.39-7.37$ (m, 5H), 7.32-7.29 (m, 1H), 6.36 (s, 1H), 3.84 (s, 2H). ${ }^{13} \mathrm{C}$ NMR (126 MHz, $\left.\mathrm{CDCl}_{3}\right): \delta$ 192.4, 137.8, 137.2, 136.0, 133.3, 130.9, 130.5, 130.4, 128.9, 128.9, 113.3, 40.5. IR (neat): 2924, 2851, 2111, 1693, 1630, 1595. HRMS (ESI+): $m / z\left(\mathrm{M}-\mathrm{N}_{2}+\mathrm{H}\right)^{+}$calculated for $\mathrm{C}_{16} \mathrm{H}_{14} \mathrm{ON} 236.1075$, found 236.1086 .

(Z)-2-(2-Azido-3-phenylprop-1-enyl)-5-methoxybenzaldehyde (3n). 84 mg (72\%). Colorless oil, $\mathrm{R}_{f}=0.30$ (EtOAc/Hexane 10:90). ${ }^{1} \mathrm{H}$ NMR (400 MHz, $\left.\mathrm{CDCl}_{3}\right): \delta 10.12(\mathrm{~s}, 1 \mathrm{H})$, $7.56(\mathrm{~d}, J=8.6 \mathrm{~Hz}, 1 \mathrm{H}), 7.40-7.34(\mathrm{~m}, 5 \mathrm{H}), 7.31(\mathrm{~d}, J=4.3 \mathrm{~Hz}, 1 \mathrm{H}), 7.11(\mathrm{dd}, J=8.6,2.9$ $\mathrm{Hz}, 1 \mathrm{H}), 6.21(\mathrm{~s}, 1 \mathrm{H}), 3.86(\mathrm{~s}, 3 \mathrm{H}), 3.83(\mathrm{~s}, 2 \mathrm{H}) .{ }^{13} \mathrm{C}$ NMR $\left(101 \mathrm{MHz}, \mathrm{CDCl}_{3}\right): \delta 191.8$, $158.8,137.0,136.1,134.1,131.8,130.4,128.9,128.8,127.3,120.6,112.8,112.6,55.5,40.3$. 


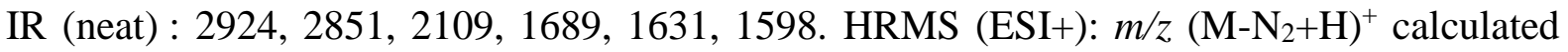
for $\mathrm{C}_{17} \mathrm{H}_{16} \mathrm{O}_{2} \mathrm{~N} 266.1181$, found 266.1192 .

(Z)-2-(2-Azido-3-phenylprop-1-enyl)-4,5-dimethoxybenzaldehyde (3o). $108 \mathrm{mg}$ (91\%). Colorless oil, $\mathrm{R}_{f}=0.40\left(\right.$ EtOAc/Hexane 20:80). ${ }^{1} \mathrm{H}$ NMR $\left(500 \mathrm{MHz}, \mathrm{CDCl}_{3}\right): \delta 10.06(\mathrm{~s}, 1 \mathrm{H})$, 7.42-7.35 (m, 5H), 7.34-7.31 (m, 1H), $7.11(\mathrm{~s}, 1 \mathrm{H}), 6.22(\mathrm{~s}, 1 \mathrm{H}), 3.96(\mathrm{~s}, 3 \mathrm{H}), 3.93(\mathrm{~s}, 3 \mathrm{H})$, 3.83 (s, 2H). ${ }^{13} \mathrm{C}$ NMR (126 MHz, $\left.\mathrm{CDCl}_{3}\right): \delta$ 190.2, 153.3, 148.3, 137.6, 135.9, 132.8, 129.0, 128.9, 127.4, 126.6, 112.2, 112.0, 110.4, 56.2, 56.0, 40.4. IR (neat): 2926, 2851, 2118, 2086, 1677, 1632, 1594. HRMS (ESI+): $m / z\left(M-\mathrm{N}_{2}+\mathrm{H}\right)^{+}$calculated for $\mathrm{C}_{18} \mathrm{H}_{18} \mathrm{O}_{3} \mathrm{~N} 296.1287$, found 296.1297.

(Z)-2-(2-Azido-5-(tert-butyldimethylsilyloxy)pent-1-enyl)benzaldehyde (3p). $97 \mathrm{mg}$ (80\%). Colorless oil, $\mathrm{R}_{f}=0.10$ (EtOAc/Hexane 10:90). ${ }^{1} \mathrm{H}$ NMR (400 MHz, $\left.\mathrm{CDCl}_{3}\right): \delta 10.09$ (s, 1H), $7.78(\mathrm{dd}, J=7.7,1.1 \mathrm{~Hz}, 1 \mathrm{H}), 7.61-7.55(\mathrm{~m}, 1 \mathrm{H}), 7.48(\mathrm{dt}, J=13.4,2.9 \mathrm{~Hz}, 1 \mathrm{H}), 7.32(\mathrm{t}, J=$ $7.2 \mathrm{~Hz}, 1 \mathrm{H}), 6.25(\mathrm{~s}, 1 \mathrm{H}), 3.70(\mathrm{t}, J=6.0 \mathrm{~Hz}, 2 \mathrm{H}), 2.70-2.32(\mathrm{~m}, 2 \mathrm{H}), 1.92-1.71(\mathrm{~m}, 2 \mathrm{H}), 0.86$ (s, 9H), 0.03 (s, 6H). ${ }^{13} \mathrm{C}$ NMR (101 MHz, $\left.\mathrm{CDCl}_{3}\right): \delta 192.3,139.1,137.6,133.3,133.0$, 130.5, 130.3, 127.1, 111.1, 61.6, 31.0, 30.1, 26.0 25.9, -5.3. HRMS (ESI+): $\mathrm{m} / \mathrm{z}\left(\mathrm{M}-\mathrm{N}_{2}+\mathrm{H}\right)^{+}$ calculated for $\mathrm{C}_{18} \mathrm{H}_{28} \mathrm{NO}_{2} \mathrm{Si} 318.1889$, found 318.1890 .

(Z)-2-(2-Azido-5-(tert-butyldimethylsilyloxy)pent-1-enyl)-5-(tert-butyldimethylsilyloxy)4-methoxybenzaldehyde (3q). $108 \mathrm{mg}(84 \%)$. Colorless oil, $\mathrm{R}_{f}=0.15$ (EtOAc/Hexane 10:90). ${ }^{1} \mathrm{H}$ NMR (400 MHz, $\mathrm{CDCl}_{3}$ ): $\delta 9.99$ (s, 1H), 7.29 (s, 1H), 7.13 (s, 1H), 6.30 (s, 1H), 3.89 (s, $3 \mathrm{H}), 3.75(\mathrm{t}, J=6.0 \mathrm{~Hz}, 2 \mathrm{H}), 2.62-2.50(\mathrm{~m}, 2 \mathrm{H}), 1.91-1.84(\mathrm{~m}, 2 \mathrm{H}), 1.00(\mathrm{~s}, 9 \mathrm{H}), 0.92(\mathrm{~s}, 9 \mathrm{H})$, 0.17 (s, 6H), $0.08(\mathrm{~s}, 6 \mathrm{H}) .{ }^{13} \mathrm{C}$ NMR $\left(101 \mathrm{MHz}, \mathrm{CDCl}_{3}\right): \delta$ 190.7, 155.3, 144.1, 138.1, 133.0, 126.7, 121.6, 112.6, 110.8, 61.7, 55.6, 31.0, 30.2, 25.9, 25.6, 18.4, 18.3, -4.5, -5.2. IR (neat): 2954, 2930, 2857, 2112, 1688, 1599. HRMS (ESI+): $m / z\left(\mathrm{M}-\mathrm{N}_{2}+\mathrm{H}\right)^{+}$calculated for $\mathrm{C}_{25} \mathrm{H}_{44} \mathrm{O}_{4} \mathrm{NSi}_{2} 478.2809$, found 478.2837 .

(Z)-2-(2-Azidohex-1-enyl)-5-fluorobenzaldehyde (3r). $96 \mathrm{mg}(86 \%)$. Colorless oil, $\mathrm{R}_{f}=$ 0.10 (EtOAc/Hexane 10:90). ${ }^{1} \mathrm{H}$ NMR (400 MHz, $\left.\mathrm{CDCl}_{3}\right): \delta 10.11(\mathrm{~d}, J=2.3 \mathrm{~Hz}, 1 \mathrm{H}), 7.57$ (dd, $J=8.6,5.3 \mathrm{~Hz}, 1 \mathrm{H}), 7.52(\mathrm{dd}, J=8.8,2.9 \mathrm{~Hz}, 1 \mathrm{H}), 7.28-7.19(\mathrm{~m}, 1 \mathrm{H}), 6.14(\mathrm{~s}, 1 \mathrm{H})$, 2.54-2.41 (m, 2H), 1.68-1.64 (m, 2H), 1.51-1.46 (m, 2H), $1.00(\mathrm{t}, J=7.3 \mathrm{~Hz}, 3 \mathrm{H}) .{ }^{13} \mathrm{C}$ NMR $\left(101 \mathrm{MHz}, \mathrm{CDCl}_{3}\right): \delta$ 190.8, 162.7, 139.8, $134.6(\mathrm{~d}, J=5.7 \mathrm{~Hz}), 134.0(\mathrm{~d}, J=3.0 \mathrm{~Hz}), 132.4$ (d, $J=7.0 \mathrm{~Hz}), 120.6$ (d, $J=21 . \mathrm{Hz}), 115.61(\mathrm{~d}, J=22 . \mathrm{Hz}), 109.7,33.3,29.9,22.1,13.8 . \mathrm{IR}$ (neat): 2958, 2930, 2862, 2112, 1694, 1638, 1592. HRMS (ESI+): $m / z\left(\mathrm{M}-\mathrm{N}_{2}+\mathrm{H}\right)^{+}$calculated for $\mathrm{C}_{13} \mathrm{H}_{15} \mathrm{ONF} 220.1138$, found 220.1147 . 
(Z)-2-(5-Azidooct-4-en-4-yl)benzaldehyde (3s). $99 \mathrm{mg}$ (84\%). Colorless oil, $\mathrm{R}_{f}=0.10$ (EtOAc/Hexane 10:90). ${ }^{1} \mathrm{H}$ NMR (500 MHz, $\mathrm{CDCl}_{3}$ ): $\delta 10.03$ (s, 1H), $7.94(\mathrm{~d}, J=7.7 \mathrm{~Hz}$, 1H), $7.58(\mathrm{dd}, J=10.8,4.2 \mathrm{~Hz}, 1 \mathrm{H}), 7.40$ (t, $J=7.5 \mathrm{~Hz}, 1 \mathrm{H}), 7.16(\mathrm{~d}, J=7.7 \mathrm{~Hz}, 1 \mathrm{H}), 2.60$ $2.45(\mathrm{~m}, 2 \mathrm{H}), 2.43-2.29(\mathrm{~m}, 2 \mathrm{H}), 1.72-1.62(\mathrm{~m}, 2 \mathrm{H}), 1.32-1.27(\mathrm{~m}, 2 \mathrm{H}), 1.08(\mathrm{t}, J=7.4 \mathrm{~Hz}$, $3 \mathrm{H}), 0.88(\mathrm{t}, J=7.3 \mathrm{~Hz}, 3 \mathrm{H}) .{ }^{13} \mathrm{C} \mathrm{NMR}\left(126 \mathrm{MHz}, \mathrm{CDCl}_{3}\right): \delta 192.5,159.2,156.6,133.6$, 129.3, 127.4, 123.3, 122.1, 121.9, 36.5, 30.4, 21.8, 21.6, 14.1, 13.7. HRMS (ESI+): $\mathrm{m} / z$ (M$\left.\mathrm{N}_{2}+\mathrm{H}\right)^{+}$calculated for $\mathrm{C}_{15} \mathrm{H}_{20} \mathrm{ON} 230.1545$, found 230.1550 .

(Z)-Methyl 2-(2-azidohex-1-enyl)benzoate (3t). $89 \mathrm{mg}$ (79\%). Colorless oil, $\mathrm{R}_{f}=0.10$ (EtOAc/Hexane 10:90). ${ }^{1} \mathrm{H}$ NMR (400 MHz, $\left.\mathrm{CDCl}_{3}\right): \delta 7.89(\mathrm{dd}, J=7.9,1.3 \mathrm{~Hz}, 1 \mathrm{H}), 7.73$ (d, $J=7.9 \mathrm{~Hz}, 1 \mathrm{H}), 7.47$ (td, $J=7.6,1.3 \mathrm{~Hz}, 1 \mathrm{H}), 7.29-7.22(\mathrm{~m}, 1 \mathrm{H}), 6.33$ (s, 1H), 3.87 (s, $3 \mathrm{H}), 2.50-2.42(\mathrm{~m}, 2 \mathrm{H}), 1.65(\mathrm{ddd}, J=12.6,8.5,6.3 \mathrm{~Hz}, 2 \mathrm{H}), 1.48(\mathrm{dd}, J=14.9,7.4 \mathrm{~Hz}, 2 \mathrm{H})$, $0.99(\mathrm{t}, J=7.3 \mathrm{~Hz}, 3 \mathrm{H}) .{ }^{13} \mathrm{C} \mathrm{NMR}\left(126 \mathrm{MHz}, \mathrm{CDCl}_{3}\right): \delta 167.8,136.4,136.3,131.5,130.7$, 130.3, 128.7, 126.5, 114.0, 51.9, 33.5, 30.0, 22.0, 13.8. IR (neat): 2956, 2868, 2109, 1721, 1638, 1598, 1568. HRMS (ESI+): $m / z\left(\mathrm{M}-\mathrm{N}_{2}+\mathrm{H}\right)^{+}$calculated for $\mathrm{C}_{14} \mathrm{H}_{18} \mathrm{NO}_{2} 232.1338$, found 232.1345

(Z)-1-(2-(2-Azidohex-1-enyl)phenyl)ethanone(3u). $93 \mathrm{mg}$ (84\%). Colorless oil, $\mathrm{R}_{f}=0.10$ (EtOAc/Hexane 10:90). ${ }^{1} \mathrm{H}$ NMR (500 MHz, $\left.\mathrm{CDCl}_{3}\right): \delta 7.79-7.54(\mathrm{~m}, 2 \mathrm{H}), 7.44(\mathrm{td}, J=7.8$, $1.2 \mathrm{~Hz}, 1 \mathrm{H}), 7.28(\mathrm{t}, J=7.6 \mathrm{~Hz}, 1 \mathrm{H}), 6.12(\mathrm{~s}, 1 \mathrm{H}), 2.56(\mathrm{~s}, 3 \mathrm{H}), 2.43(\mathrm{dd}, J=9.9,5.4 \mathrm{~Hz}, 2 \mathrm{H})$, 1.71-1.55 (m, 2H), 1.52-1.42 (m, 2H), $0.98(\mathrm{t}, J=7.3 \mathrm{~Hz}, 3 \mathrm{H}) .{ }^{13} \mathrm{C} \mathrm{NMR}\left(101 \mathrm{MHz}, \mathrm{CDCl}_{3}\right)$ : $\delta 201.8,137.5,136.6,134.4,131.0,130.8,128.7,126.6,114.0,33.4,29.9,29.6,22.1,13.8$. IR (neat): 2958, 2930, 2867, 2110, 1683, 1638, 1595, 1565. HRMS (ESI+): $m / z\left(\mathrm{M}^{\left.-\mathrm{N}_{2}+\mathrm{H}\right)^{+}}\right.$ calculated for $\mathrm{C}_{14} \mathrm{H}_{18} \mathrm{ON} 216.1382$, found 216.1396.

(Z)-2-(2-Azidohex-1-enyl)benzamide (3v). $96 \mathrm{mg} \quad(86 \%)$. Colorless oil, $\quad \mathrm{R}_{f}=0.30$ (EtOAc/Hexane 30:70). ${ }^{1} \mathrm{H}$ NMR $\left(500 \mathrm{MHz}, \mathrm{CDCl}_{3}\right): \delta 7.79(\mathrm{~d}, J=7.9 \mathrm{~Hz}, 1 \mathrm{H}), 7.55$ (dd, $J=$ 7.7, 1.3 Hz, 1H), 7.41 (td, $J=7.7,1.3 \mathrm{~Hz}, 1 \mathrm{H}), 7.26-7.22$ (m, 1H), 6.04 (s, 1H), 5.79 (br, 2H), 2.49-2.41 (m, 2H), 1.65-1.60 (m, 2H), 1.46-1.43 (m, 2H), $0.98(\mathrm{t}, J=7.3 \mathrm{~Hz}, 3 \mathrm{H}) .{ }^{13} \mathrm{C}$ NMR $\left(101 \mathrm{MHz}, \mathrm{CDCl}_{3}\right): \delta 171.1,137.6,133.2,133.0,130.1,130.1,127.5,126.8,112.4,33.6$, 30.0, 22.1, 13.8. IR (neat): 2956, 2864, 2108, 1717, 1595, 1494. IR (neat) : 3365, 3178, 2926, 2857, 2105, 1644, 1453. HRMS (ESI+): $m / z\left(M-\mathrm{N}_{2}+\mathrm{H}\right)^{+}$calculated for $\mathrm{C}_{13} \mathrm{H}_{17} \mathrm{~N}_{2} \mathrm{O} 217.1341$, found 217.1328 .

(Z)-3-(2-Azidohex-1-enyl)thiophene-2-carbaldehyde (7a). $97 \mathrm{mg}(88 \%)$. Colorless oil, $\mathrm{R}_{f}=$ $0.10\left(\right.$ EtOAc/Hexane 10:90). ${ }^{1} \mathrm{H}$ NMR $\left(500 \mathrm{MHz}, \mathrm{CDCl}_{3}\right): \delta 10.03$ (s, 1H), 7.76 (d, $J=5.2$ $\mathrm{Hz}, 1 \mathrm{H}), 7.63(\mathrm{~d}, J=5.2 \mathrm{~Hz}, 1 \mathrm{H}), 6.30(\mathrm{~s}, 1 \mathrm{H}), 2.55-2.43(\mathrm{~m}, 2 \mathrm{H}), 1.65(\mathrm{dt}, J=12.7,7.5 \mathrm{~Hz}$, 
$2 \mathrm{H}), 1.52-1.43(\mathrm{~m}, 2 \mathrm{H}), 0.99(\mathrm{t}, J=7.3 \mathrm{~Hz}, 3 \mathrm{H}) .{ }^{13} \mathrm{C} \mathrm{NMR}\left(101 \mathrm{MHz}, \mathrm{CDCl}_{3}\right): \delta$ 182.1, 143.4, 140.1, 137.3, 133.7, 130.2, 106.4, 33.8, 30.1, 22.1, 13.8. IR (neat): 2958, 2930, 2867, 2112, 1655, 1627, 1522. HRMS (ESI+): $\mathrm{m} / z\left(\mathrm{M}-\mathrm{N}_{2}+\mathrm{H}\right)^{+}$calculated for $\mathrm{C}_{11} \mathrm{H}_{14} \mathrm{NOS} 208.0796$, found 208.0807 .

(Z)-3-(2-Azido-3-cyclohexylprop-1-enyl) thiophene-2-carbaldehyde (7b). $105 \mathrm{mg}$ (92\%). Colorless oil, $\mathrm{R}_{f}=0.10$ (EtOAc/Hexane 10:90). ${ }^{1} \mathrm{H}$ NMR (400 MHz, $\left.\mathrm{CDCl}_{3}\right): \delta 10.03$ (d, $J=$ $0.8 \mathrm{~Hz}, 1 \mathrm{H}), 7.76$ (d, $J=5.2 \mathrm{~Hz}, 1 \mathrm{H}), 7.63$ (d, $J=5.2 \mathrm{~Hz}, 1 \mathrm{H}), 6.24$ (s, 1H), 2.37 (d, J= 7.2 $\mathrm{Hz}, 2 \mathrm{H}), 1.91-1.71(\mathrm{~m}, 5 \mathrm{H}), 1.62-1.59(\mathrm{~m}, 1 \mathrm{H}), 1.37-1.14(\mathrm{~m}, 3 \mathrm{H}), 1.03-1.00(\mathrm{~m}, 2 \mathrm{H}) .{ }^{13} \mathrm{C}$ NMR (101 MHz, $\left.\mathrm{CDCl}_{3}\right): \delta$ 182.1, 143.4, 138.7, 137.3, 133.7, 130.1, 107.7, 42.0, 36.7, 32.9, 26.2, 26.1. HRMS (ESI+): $m / z\left(\mathrm{M}-\mathrm{N}_{2}+\mathrm{H}\right)^{+}$calculated for $\mathrm{C}_{14} \mathrm{H}_{18} \mathrm{NOS} 248.1109$, found 248.1130 .

(Z)-3-(2-Azido-3-phenylprop-1-enyl) thiophene-2-carbaldehyde (7c). $99 \mathrm{mg}$ (87\%). Colorless oil, $\mathrm{R}_{f}=0.20\left(\right.$ EtOAc/Hexane 10:90). ${ }^{1} \mathrm{H}$ NMR $\left(500 \mathrm{MHz}, \mathrm{CDCl}_{3}\right): \delta 9.97(\mathrm{~d}, J=$ $0.6 \mathrm{~Hz}, 1 \mathrm{H}), 7.79$ (d, $J=5.2 \mathrm{~Hz}, 1 \mathrm{H}), 7.64(\mathrm{~d}, J=5.1 \mathrm{~Hz}, 1 \mathrm{H}), 7.44-7.28(\mathrm{~m}, 5 \mathrm{H}), 6.31$ (s, $1 \mathrm{H}), 3.85$ (s, 2H). ${ }^{13} \mathrm{C} \mathrm{NMR}\left(101 \mathrm{MHz}, \mathrm{CDCl}_{3}\right): \delta 182.1,143.0,138.4,137.7,135.6,133.8$, 130.1, 129.0, 128.8, 127.5, 108.2, 40.7. IR (neat): 3448, 3086, 2923, 2127, 1651, 1622. HRMS (ESI+): $m / z(\mathrm{M}+\mathrm{Na})^{+}$calculated for $\mathrm{C}_{14} \mathrm{H}_{11} \mathrm{ON}_{3} \mathrm{SNa} 292.0520$, found 292.0532.

(Z)-3-(2-Azidohex-1-enyl)benzo[b]thiophene-2-carbaldehyde (7d). 97 mg (75\%). Colourless oil, $\mathrm{R}_{f}=0.10$ (EtOAc/Hexane 10:90). ${ }^{1} \mathrm{H}$ NMR (400 MHz, $\mathrm{CDCl}_{3}$ ): $\delta 10.15$ (s, 1H), 7.88-7.84 (m, 1H), 7.80-7.77 (m, 1H), 7.52-7.47 (m, 1H), 7.42 (ddd, $J=8.1,7.1,1.1 \mathrm{~Hz}$, $1 \mathrm{H}), 5.96(\mathrm{t}, J=0.8 \mathrm{~Hz}, 1 \mathrm{H}), 2.64-2.54(\mathrm{~m}, 2 \mathrm{H}), 1.80-1.67(\mathrm{~m}, 2 \mathrm{H}), 1.60-1.48(\mathrm{~m}, 2 \mathrm{H}), 1.04$ $(\mathrm{t}, J=7.3 \mathrm{~Hz}, 3 \mathrm{H}) .{ }^{13} \mathrm{C} \mathrm{NMR}\left(126 \mathrm{MHz}, \mathrm{CDCl}_{3}\right): \delta 184.5,142.2,140.6,139.0,137.7,136.5$, $127.1,123.8,123.4,122.2,104.1,32.2,28.9,21.1,12.8 . \mathrm{HRMS}(\mathrm{ESI}+): \mathrm{m} / \mathrm{z}\left(\mathrm{M}_{-} \mathrm{N}_{2}+\mathrm{H}\right)^{+}$ calculated for $\mathrm{C}_{15} \mathrm{H}_{16} \mathrm{ONS} 258.0953$, found 258.0973.

General Procedure for the Cyclisation of Vinyl Azides 3 and 7. Synthesis of compounds $\mathbf{4 a - 4} \boldsymbol{u}$ and $\mathbf{8 a}-\mathbf{8 d}$. A solution of triethylphosphite $(58 \mathrm{mg}, 0.349 \mathrm{mmol}$ ) in THF (3 $\mathrm{mL})$ was added to a stirred solution of azide 3 or $7(0.349 \mathrm{mmol})$ in THF $(2 \mathrm{~mL})$ with $4 \AA$ molecular sieves at room temperature. Nitrogen evolved immediately. After $30 \mathrm{~min}$, an additional triethylphosphite $(116 \mathrm{mg}, 0.698 \mathrm{mmol})$ was added and the mixture was heated at $35^{\circ} \mathrm{C}$ for $1.5 \mathrm{~h}$. The solvent was removed under reduced pressure to give a residue, which was purified by column chromatography (230-400 mesh Silica gel, EtOAc in Hexane) to afford the isoquinoline 4 or thienopyridine 8. 
3-Butylisoquinoline (4a). ${ }^{16} 60 \mathrm{mg}$ (93\%). Colorless oil, $\mathrm{R}_{f}=0.40$ (EtOAc/Hexane 10:90). ${ }^{1} \mathrm{H}$ NMR (400 MHz, $\left.\mathrm{CDCl}_{3}\right): \delta 9.20(\mathrm{~s}, 1 \mathrm{H}), 7.93(\mathrm{dd}, J=8.2,0.7 \mathrm{~Hz}, 1 \mathrm{H}), 7.75(\mathrm{~d}, J=7.8$ $\mathrm{Hz}, 1 \mathrm{H}), 7.64(\mathrm{ddd}, J=8.2,6.8,1.2 \mathrm{~Hz}, 1 \mathrm{H}), 7.52(\mathrm{ddd}, J=8.1,6.9,1.1 \mathrm{~Hz}, 1 \mathrm{H}), 7.47$ (s, $1 \mathrm{H}), 2.97-2.92(\mathrm{~m}, 2 \mathrm{H}), 1.86-1.74(\mathrm{~m}, 2 \mathrm{H}), 1.49-1.38(\mathrm{~m}, 2 \mathrm{H}), 0.97(\mathrm{t}, J=7.4 \mathrm{~Hz}, 3 \mathrm{H}) .{ }^{13} \mathrm{C}$ NMR $\left(126 \mathrm{MHz}, \mathrm{CDCl}_{3}\right): \delta 155.8,152.0,136.5,130.2,127.5,127.0,126.2,126.1,117.9$, 37.8, 32.1, 22.5, 14.0. HRMS (ESI+): $m / z(\mathrm{M}+\mathrm{H})^{+}$calculated for $\mathrm{C}_{13} \mathrm{H}_{16} \mathrm{~N}$ 186.1283, found 186.1288 .

3-Butyl-7-methoxyisoquinoline (4b). $56 \mathrm{mg}$ (85\%). Colorless oil, $\mathrm{R}_{f}=0.70$ (EtOAc/Hexane 20:80). ${ }^{1} \mathrm{H}$ NMR (400 MHz, $\left.\mathrm{CDCl}_{3}\right): \delta 9.10$ (s, 1H), 7.65 (d, J = $9.0 \mathrm{~Hz}$, 1H), 7.40 (s, 1H), 7.32-7.27 (m, 1H), 7.18 (d, $J=2.5 \mathrm{~Hz}, 1 \mathrm{H}), 3.93$ (s, 3H), 2.94-2.87 (m, 2H), 1.84-1.69 (m, 2H), 1.50-1.36 (m, 2H), $0.96(\mathrm{t}, J=7.4 \mathrm{~Hz}, 3 \mathrm{H}) .{ }^{13} \mathrm{C}$ NMR $(126 \mathrm{MHz}$, $\left.\mathrm{CDCl}_{3}\right): \delta 157.7,154.0,150.5,132.2,128.0,127.6,123.4,117.8,104.5,55.4,37.6,32.2$, 22.5, 14.0. HRMS (ESI+): $m / z(\mathrm{M}+\mathrm{H})^{+}$calculated for $\mathrm{C}_{14} \mathrm{H}_{18} \mathrm{NO} 216.1388$, found 216.1389 .

3-Butyl-7-methylisoquinoline (4c). $57 \mathrm{mg}$ (85\%). Yellow solid ( $\left.\mathrm{m} \cdot \mathrm{p}=50-55{ }^{\circ} \mathrm{C}\right), \mathrm{R}_{f}=$ 0.40 (EtOAc/Hexane 10:90). ${ }^{1} \mathrm{H}$ NMR (500 MHz, $\left.\mathrm{CDCl}_{3}\right): \delta 9.13(\mathrm{~s}, 1 \mathrm{H}), 7.82(\mathrm{~d}, J=8.3 \mathrm{~Hz}$, $1 \mathrm{H}), 7.51(\mathrm{~s}, 1 \mathrm{H}), 7.37$ (s, 1H), $7.35(\mathrm{dd}, J=8.3,1.4 \mathrm{~Hz}, 1 \mathrm{H}), 2.95-2.88(\mathrm{~m}, 2 \mathrm{H}), 2.53$ (s, $1 \mathrm{H}), 1.85-1.72(\mathrm{~m}, 2 \mathrm{H}), 1.47-1.36(\mathrm{~m}, 2 \mathrm{H}), 0.96(\mathrm{t}, J=7.4 \mathrm{~Hz}, 3 \mathrm{H}) .{ }^{13} \mathrm{C}$ NMR $(126 \mathrm{MHz}$, $\left.\mathrm{CDCl}_{3}\right): \delta 155.8,151.6,140.5,136.9,128.6,127.3,125.5,125.0,117.4,37.8,32.1,22.5$, 22.1, 14.0. HRMS (ESI+): $m / z(\mathrm{M}+\mathrm{H})^{+}$calculated for $\mathrm{C}_{14} \mathrm{H}_{18} \mathrm{~N} 200.1439$, found 200.1447.

2-Butylbenzo[f]isoquinoline (4d). $56 \mathrm{mg}(83 \%)$. Colorless oil, $\mathrm{R}_{f}=0.40($ EtOAc/Hexane 10:90). ${ }^{1} \mathrm{H}$ NMR (500 MHz, $\left.\mathrm{CDCl}_{3}\right): \delta 9.17$ (s, 1H), 8.68 (ddd, $\left.J=7.0,3.3,3.2 \mathrm{~Hz}, 1 \mathrm{H}\right), 8.26$ (s, 1H), $7.91(\mathrm{dt}, J=5.7,3.1 \mathrm{~Hz}, 1 \mathrm{H}), 7.77(\mathrm{~s}, 2 \mathrm{H}), 7.74-7.64(\mathrm{~m}, 2 \mathrm{H}), 3.11-2.99(\mathrm{~m}, 2 \mathrm{H})$, 1.94-1.81 (m, 2H), 1.54-1.42 (m, 2H), 0.99 (t, $\left.J=7.4 \mathrm{~Hz}, 3 \mathrm{H}) .{ }^{13} \mathrm{C} \mathrm{NMR} \mathrm{(126} \mathrm{MHz,} \mathrm{CDCl}_{3}\right)$ : $\delta 158.1,151.2,135.4,133.6,128.7,128.5,128.4,127.3,126.9,125.0,124.7,123.1,114.1$, 38.4, 32.5, 22.6, 14.0. HRMS (ESI+): $m / z(\mathrm{M}+\mathrm{H})^{+}$calculated for $\mathrm{C}_{17} \mathrm{H}_{18} \mathrm{~N} 236.1439$, found 236.1441 .

3-Butyl-6,7-dimethoxyisoquinoline (4e). ${ }^{16} 57 \mathrm{mg}$ (84\%). Yellow solid (m.p = 160-162 $\left.{ }^{\circ} \mathrm{C}\right), \mathrm{R}_{f}=0.50\left(\right.$ EtOAc/Hexane 40:60). ${ }^{1} \mathrm{H}$ NMR (400 MHz, $\left.\mathrm{CDCl}_{3}\right): \delta 9.29(\mathrm{~s}, 1 \mathrm{H}), 7.68(\mathrm{~s}$, 1H), 7.51 (s, 1H), 7.19 (s, 1H), 4.13 (s, 3H), 4.10 (s, 3H), 3.22 (dd, J = 7.6, 7.6 Hz, 2H), 1.98$1.85(\mathrm{~m}, 2 \mathrm{H}), 1.51-1.41(\mathrm{~m}, 2 \mathrm{H}), 0.98(\mathrm{t}, J=7.3 \mathrm{~Hz}, 3 \mathrm{H}) .{ }^{13} \mathrm{C} \mathrm{NMR}\left(126 \mathrm{MHz}, \mathrm{CDCl}_{3}\right): \delta$ 157.8, 152.4, 145.9, 142.0, 137.4, 122.6, 120.6, 106.6, 104.4, 56.8 (2C), 32.4, 31.4, 22.0, 13.7. HRMS (ESI+): $m / z(\mathrm{M}+\mathrm{H})^{+}$calculated for $\mathrm{C}_{15} \mathrm{H}_{20} \mathrm{O}_{2} \mathrm{~N} 246.1490$, found 246.1504 .

3-Butyl-6-(tertbutyldimethylsilyloxy)-7-methoxyisoquinoline (4f). $64 \mathrm{mg}$ (90\%). Yellow solid (m.p $\left.=116-119{ }^{\circ} \mathrm{C}\right), \mathrm{R}_{f}=0.50\left(\right.$ EtOAc/Hexane 10:90). ${ }^{1} \mathrm{H}$ NMR $\left(400 \mathrm{MHz}, \mathrm{CDCl}_{3}\right): \delta$ 
$8.93(\mathrm{~s}, 1 \mathrm{H}), 7.32(\mathrm{~s}, 1 \mathrm{H}), 7.25(\mathrm{~s}, 1 \mathrm{H}), 6.97(\mathrm{~s}, 1 \mathrm{H}), 3.94(\mathrm{~s}, 1 \mathrm{H}), 2.94-2.81(\mathrm{~m}, 2 \mathrm{H}), 1.83-$ $1.68(\mathrm{~m}, 2 \mathrm{H}), 1.48-1.34(\mathrm{~m}, 2 \mathrm{H}), 1.03(\mathrm{~s}, 9 \mathrm{H}), 0.96(\mathrm{t}, J=7.4 \mathrm{~Hz}, 3 \mathrm{H}), 0.20(\mathrm{~s}, 6 \mathrm{H}) .{ }^{13} \mathrm{C}$ NMR (126 MHz, $\left.\mathrm{CDCl}_{3}\right): \delta 155.2,154.3,149.5,145.8,133.9,123.0,116.9,115.4,104.3$, 55.4, 37.6, 32.3, 25.7, 22.5, 18.5, 14.0, -4.6. HRMS (ESI+): $\mathrm{m} / \mathrm{z}(\mathrm{M}+\mathrm{H})^{+}$calculated for $\mathrm{C}_{20} \mathrm{H}_{32} \mathrm{O}_{2} \mathrm{NSi}$ 346.2202, found 346.2200.

8-Butyl-[1,3]dioxolo[4,5-f]isoquinoline (4g). $56 \mathrm{mg}$ (82\%). Colorless oil, $\mathrm{R}_{f}=0.50$ (EtOAc/Hexane 20:80). ${ }^{1} \mathrm{H}$ NMR (500 MHz, $\mathrm{CDCl}_{3}$ ): $\delta 9.25$ (s, 1H), 7.39 (s, 1H), 7.35-7.31 $(\mathrm{m}, 2 \mathrm{H}), 6.21(\mathrm{~s}, 2 \mathrm{H}), 3.10-2.72(\mathrm{~m}, 2 \mathrm{H}), 1.87-1.71(\mathrm{~m}, 2 \mathrm{H}), 1.47-1.35(\mathrm{~m}, 2 \mathrm{H}), 0.96(\mathrm{t}, J=$ $7.4 \mathrm{~Hz}, 3 \mathrm{H}) .{ }^{13} \mathrm{C} \mathrm{NMR}\left(126 \mathrm{MHz}, \mathrm{CDCl}_{3}\right): \delta 153.6,145.2,143.8,132.4,119.5,117.9,114.8$, 113.8, 102.2, 37.7, 32.1, 22.5, 14.0. HRMS (ESI $m / z(\mathrm{M}+\mathrm{H})^{+}$calculated for $\mathrm{C}_{14} \mathrm{H}_{16} \mathrm{O}_{2} \mathrm{~N}$ 230,1181 , found 230.1192 .

3-(Cyclohexylmethyl)isoquinoline (4h). $61 \mathrm{mg}(91 \%)$. Yellow solid (m.p $\left.=45-48{ }^{\circ} \mathrm{C}\right), \mathrm{R}_{f}$ $=0.40\left(\right.$ EtOAc/Hexane 10:90) ${ }^{1} \mathrm{H}$ NMR $\left(500 \mathrm{MHz}, \mathrm{CDCl}_{3}\right): \delta 9.15(\mathrm{~s}, 1 \mathrm{H}), 7.86(\mathrm{~d}, J=8.2$ $\mathrm{Hz}, 1 \mathrm{H}), 7.68(\mathrm{~d}, J=8.2 \mathrm{~Hz}, 1 \mathrm{H}), 7.62-7.54(\mathrm{~m}, 1 \mathrm{H}), 7.46$ (t, $J=7.5 \mathrm{~Hz}, 1 \mathrm{H}), 7.36(\mathrm{~s}, 1 \mathrm{H})$, $2.74(\mathrm{~d}, J=7.1 \mathrm{~Hz}, 2 \mathrm{H}), 1.89-1.73(\mathrm{~m}, 1 \mathrm{H}), 1.66-1.57$ (m, 5H), 1.28-1.05 (m, 4H), 1.03-0.89 $(\mathrm{m}, 2 \mathrm{H}) .{ }^{13} \mathrm{C} \mathrm{NMR}\left(126 \mathrm{MHz}, \mathrm{CDCl}_{3}\right): \delta 154.5,152.0,136.3,130.2,127.5,127.0,126.3$, 126.0, 119.0, 46.0, 38.5, 33.2, 26.6, 26.3. HRMS (ESI+): $\mathrm{m} / \mathrm{z}(\mathrm{M}+\mathrm{H})^{+}$calculated for $\mathrm{C}_{16} \mathrm{H}_{20} \mathrm{~N}$ 226.1596, found 226.1595.

3-(Cyclohexylmethyl)-7-methoxyisoquinoline (4i). $61 \mathrm{mg}(89 \%)$. Colorless oil, $\mathrm{R}_{f}=0.35$ (EtOAc/Hexane 20:80). ${ }^{1} \mathrm{H}$ NMR (500 MHz, $\left.\mathrm{CDCl}_{3}\right): \delta 9.11(\mathrm{~s}, 1 \mathrm{H}), 7.65(\mathrm{~d}, J=9.0 \mathrm{~Hz}$, 1H), $7.36(\mathrm{~s}, 1 \mathrm{H}), 7.31$ (dd, $J=8.9,2.5 \mathrm{~Hz}, 1 \mathrm{H}), 7.19$ (d, $J=2.5 \mathrm{~Hz}, 1 \mathrm{H}), 3.94$ (s, 3H), 2.77 (d, $J=7.1 \mathrm{~Hz}, 2 \mathrm{H}), 1.87-1.82(\mathrm{~m}, 1 \mathrm{H}), 1.73-1.63(\mathrm{~m}, 5 \mathrm{H}), 1.29-1.14$ (m, 3H), 1.07-0.96 (m, $2 \mathrm{H}) .{ }^{13} \mathrm{C}$ NMR $\left(126 \mathrm{MHz}, \mathrm{CDCl}_{3}\right): \delta 157.7,152.6,150.5,132.0,128.0,127.6,123.5,118.9$, 104.5, 55.4, 45.8, 38.6, 33.2, 26.6, 26.3. HRMS (ESI+): $\mathrm{m} / z(\mathrm{M}+\mathrm{H})^{+}$calculated for $\mathrm{C}_{17} \mathrm{H}_{22} \mathrm{ON}$ 256.1701, found 256.1704 .

2-(Cyclohexylmethyl)benzo[f]isoquinoline (4j). $64 \mathrm{mg}$ (92\%). Colorless oil, $\mathrm{R}_{f}=0.40$ (EtOAc/Hexane 10:90). ${ }^{1} \mathrm{H}$ NMR (400 MHz, $\mathrm{CDCl}_{3}$ ): $\delta 9.20$ (s, 1H), 8.73-8.62 (m, 1H), 8.22 (s, 1H), 7.99-7.86 (m, 1H), $7.78(\mathrm{~s}, 2 \mathrm{H}), 7.74-7.65(\mathrm{~m}, 2 \mathrm{H}), 2.92$ (d, J = 7.2 Hz, 2H), 1.97$1.87(\mathrm{~m}, 1 \mathrm{H}), 1.80-1.63(\mathrm{~m}, 5 \mathrm{H}), 1.35-1.18(\mathrm{~m}, 3 \mathrm{H}), 1.14-1.02(\mathrm{~m}, 2 \mathrm{H}) .{ }^{13} \mathrm{C}$ NMR $(126 \mathrm{MHz}$, $\left.\mathrm{CDCl}_{3}\right): \delta 156.9,151.2,135.2,133.6,128.7,128.5,128.4,127.3,126.9,125.0,124.7,123.2$, 115.1, 46.6, 38.9, 33.3, 26.6, 26.3. HRMS (ESI+): $m / z(\mathrm{M}+\mathrm{H})^{+}$calculated for $\mathrm{C}_{20} \mathrm{H}_{22} \mathrm{~N}$ 276.1752 , found 276.1752 . 
8-(Cyclohexylmethyl)-[1,3]dioxolo[4,5-f]isoquinoline (4k). $60.5 \mathrm{mg}$ (88\%). Colorless oil, $\mathrm{R}_{f}=0.40$ (EtOAc/Hexane 20:80). ${ }^{1} \mathrm{H}$ NMR (500 MHz, $\left.\mathrm{CDCl}_{3}\right): \delta 9.25(\mathrm{~s}, 1 \mathrm{H}), 7.34(\mathrm{~s}, 1 \mathrm{H})$, 7.34-7.28 (m, 2H), 6.20 (s, 2H), $2.75(\mathrm{~d}, J=7.1 \mathrm{~Hz}, 2 \mathrm{H}), 1.91-1.80(\mathrm{~m}, 1 \mathrm{H}), 1.75-1.64$ (m, $5 \mathrm{H}), 1.22-1.15(\mathrm{~m}, 3 \mathrm{H}), 1.05-0.98(\mathrm{~m}, 2 \mathrm{H}) .{ }^{13} \mathrm{C} \mathrm{NMR}\left(101 \mathrm{MHz}, \mathrm{CDCl}_{3}\right): \delta 152.2,145.1$, 143.8, 141.6, 132.2, 119.6, 119.0, 114.8, 113.8, 102.3, 46.0, 38.4, 33.2, 26.6, 26.3. HRMS (ESI+): $m / z(\mathrm{M}+\mathrm{H})^{+}$calculated for $\mathrm{C}_{17} \mathrm{H}_{20} \mathrm{ON}_{2} 270.1494$, found 270.1492 .

6-(tertButyldimethylsilyloxy)-3-(cyclohexylmethyl)-7-methoxyisoquinoline (4l). $65 \mathrm{mg}$ (90\%). Colorless oil, $\mathrm{R}_{f}=0.50$ (EtOAc/Hexane 10:90). ${ }^{1} \mathrm{H}$ NMR $\left(500 \mathrm{MHz}, \mathrm{CDCl}_{3}\right) \delta 8.94$ (s, 1H), $7.27(\mathrm{~s}, 1 \mathrm{H}), 7.24(\mathrm{~s}, 1 \mathrm{H}), 6.97(\mathrm{~s}, 1 \mathrm{H}), 3.94(\mathrm{~s}, 3 \mathrm{H}), 2.73$ (d, J = 7.1 Hz, 2H), 1.90$1.77(\mathrm{~m}, 1 \mathrm{H}), 1.71-1.63(\mathrm{~m}, 5 \mathrm{H}), 1.28-1.14(\mathrm{~m}, 3 \mathrm{H}), 1.07-0.96(\mathrm{~m}, 2 \mathrm{H}), 1.04(\mathrm{~s}, 9 \mathrm{H}), 0.20(\mathrm{~s}$, $6 \mathrm{H}) .{ }^{13} \mathrm{C} \mathrm{NMR}\left(75 \mathrm{MHz}, \mathrm{CDCl}_{3}\right): \delta 155.2,153.0,149.6,145.8,133.6,123.05,117.8,115.4$, 104.3, 55.4, 46.0, 38.5, 33.3, 26.6, 26.3, 25.7, 18.5, -4.6. HRMS (ESI+): $m / z(\mathrm{M}+\mathrm{H})^{+}$ calculated for $\mathrm{C}_{23} \mathrm{H}_{36} \mathrm{O}_{2} \mathrm{NSi} 386.2515$, found 386.2536.

3-Benzylisoquinoline (4m). ${ }^{17} 59 \mathrm{mg}$ (89\%). White solid (m.p $=56-58{ }^{\circ} \mathrm{C}$ ), $\mathrm{R}_{f}=0.30$ (EtOAc/Hexane 20:80). ${ }^{1} \mathrm{H}$ NMR (400 MHz, $\left.\mathrm{CDCl}_{3}\right): \delta 9.22$ (s, 1H), 7.93 (d, $J=8.1 \mathrm{~Hz}$, $1 \mathrm{H}), 7.71(\mathrm{~d}, J=8.2 \mathrm{~Hz}, 1 \mathrm{H}), 7.63(\mathrm{ddd}, J=8.2,6.9,1.2 \mathrm{~Hz}, 1 \mathrm{H}), 7.53$ (ddd, $J=8.0,6.9,1.1$ $\mathrm{Hz}, 1 \mathrm{H}), 7.42(\mathrm{~s}, 1 \mathrm{H}), 7.36-7.29(\mathrm{~m}, 4 \mathrm{H}), 7.27-7.19(\mathrm{~m}, 1 \mathrm{H}), 4.32$ (s, 2H). ${ }^{13} \mathrm{C}$ NMR (101 $\left.\mathrm{MHz}, \mathrm{CDCl}_{3}\right): \delta 154.4,152.3,139.8,136.5,130.4,129.2,128.6,127.5,127.1,126.6,126.3$, 126.2, 118.7, 44.3. HRMS (ESI+): $\mathrm{m} / z(\mathrm{M}+\mathrm{H})^{+}$calculated for $\mathrm{C}_{16} \mathrm{H}_{14} \mathrm{~N} 220.1126$, found 220.1133 .

3-Benzyl-7-methoxyisoquinoline (4n). $59 \mathrm{mg}(87 \%)$. White solid (m.p $=78-80{ }^{\circ} \mathrm{C}$ ), $\mathrm{R}_{f}=0.70$ (EtOAc/Hexane 40:60). ${ }^{1} \mathrm{H}$ NMR (500 MHz, $\left.\mathrm{CDCl}_{3}\right): \delta 9.12(\mathrm{~s}, 1 \mathrm{H}), 7.62(\mathrm{~d}, J=9.0 \mathrm{~Hz}$, 1H), $7.36(\mathrm{~s}, 1 \mathrm{H}), 7.33-7.28(\mathrm{~m}, 4 \mathrm{H}), 7.28(\mathrm{dd}, J=5.0,1.6 \mathrm{~Hz}, 1 \mathrm{H}), 7.22(\mathrm{dt}, J=8.8,4.5 \mathrm{~Hz}$, $1 \mathrm{H}), 7.19(\mathrm{~d}, J=2.5 \mathrm{~Hz}, 1 \mathrm{H}), 4.29$ (s, 2H), 3.93 (s, 3H). ${ }^{13} \mathrm{C} \mathrm{NMR}\left(101 \mathrm{MHz}, \mathrm{CDCl}_{3}\right): \delta$ 158.0, 152.5, 150.8, 140.0, 132.2, 129.2, 128.5, 128.2, 127.83, 126.3, 123.6, 118.6, 104.6, 55.4, 44.1. HRMS (ESI+): $m / z(\mathrm{M}+\mathrm{H})^{+}$calculated for $\mathrm{C}_{17} \mathrm{H}_{16} \mathrm{NO} 250.1232$, found 250.1248 .

3-Benzyl-6,7-dimethoxyisoquinoline (4o). ${ }^{18} 59 \mathrm{mg}(86 \%)$. Yellow solid (m.p = 182-185 ${ }^{\circ} \mathrm{C}$ ), $\mathrm{R}_{f}=0.50$ (EtOAc/Hexane 50:50). ${ }^{1} \mathrm{H}$ NMR (400 MHz, $\left.\mathrm{CDCl}_{3}\right): \delta 9.36$ (br, 1H), 7.57 (br, 1H), 7.47 (s, 1H), 7.43 (d, $J=7.2 \mathrm{~Hz}, 2 \mathrm{H}), 7.38$ (t, $J=7.4 \mathrm{~Hz}, 2 \mathrm{H}), 7.31$ (t, $J=7.1 \mathrm{~Hz}, 1 \mathrm{H})$, $7.10(\mathrm{~s}, 1 \mathrm{H}), 4.62(\mathrm{~s}, 2 \mathrm{H}), 4.09$ (s, 3H), 4.07 (s, 3H). ${ }^{13} \mathrm{C} \mathrm{NMR}\left(101 \mathrm{MHz}, \mathrm{CDCl}_{3}\right): \delta$ 157.9, 152.5, 145.0, 141.7, 137.4, 135.9, 129.6, 129.21, 127.5, 122.7, 121.1, 106.7, 104.6, 56.91, 56.8, 38.5. HRMS (ESI+): $m / z(\mathrm{M}+\mathrm{H})^{+}$calculated for $\mathrm{C}_{18} \mathrm{H}_{18} \mathrm{O}_{2} \mathrm{~N} 280.1337$, found 280.1347. 
3-(3-(tertButyldimethylsilyloxy)propyl)isoquinoline (4p). $59 \mathrm{mg}(85 \%)$. Colorless oil, $\mathrm{R}_{f}$ $=0.50\left(\right.$ EtOAc/Hexane 10:90). ${ }^{1} \mathrm{H}$ NMR $\left(500 \mathrm{MHz}, \mathrm{CDCl}_{3}\right): \delta 9.15(\mathrm{~s}, 1 \mathrm{H}), 7.88(\mathrm{~d}, J=8.1$ Hz, 1H), 7.69 (d, J= 8.2 Hz, 1H), 7.63-7.56 (m, 1H), 7.51-7.45 (m, 1H), $7.44(\mathrm{~s}, 1 \mathrm{H}), 3.65(\mathrm{t}$, $J=6.3 \mathrm{~Hz}, 2 \mathrm{H}), 2.99-2.92(\mathrm{~m}, 2 \mathrm{H}), 2.01-1.97(\mathrm{~m}, 4 \mathrm{H}), 0.86(\mathrm{~s}, 9 \mathrm{H}), 0.00(\mathrm{~s}, 6 \mathrm{H}) .{ }^{13} \mathrm{C} \mathrm{NMR}$ $\left(126 \mathrm{MHz}, \mathrm{CDCl}_{3}\right): \delta 155.2,152.0,136.5,130.2,127.5,127.1,126.3,126.1,118.1,62.5$, 34.4, 32.8, 26.1, 18.3, -5.2. HRMS (ESI+): $m / z(\mathrm{M}+\mathrm{H})^{+}$calculated for $\mathrm{C}_{18} \mathrm{H}_{28} \mathrm{ONSi} 302.1940$, found 302.1943 .

\section{6-(tertButyldimethylsilyloxy)-3-(3-(tert-butyldimethylsilyloxy)propyl)-7-}

methoxyisoquinoline (4q). $67 \mathrm{mg}(92 \%)$. Colorless oil, $\mathrm{R}_{f}=0.60\left(\right.$ EtOAc/Hexane 20:80). ${ }^{1} \mathrm{H}$ NMR (300 MHz, $\left.\mathrm{CDCl}_{3}\right): \delta 8.93$ (s, 1H), 7.34 (s, 1H), 7.25 (s, 1H), 6.97 (s, 1H), 3.95 (s, 3H), $3.70(\mathrm{t}, J=6.3 \mathrm{~Hz}, 2 \mathrm{H}), 3.04-2.82(\mathrm{~m}, 2 \mathrm{H}), 2.10-1.94(\mathrm{~m}, 2 \mathrm{H}), 1.04(\mathrm{~s}, 9 \mathrm{H}), 0.91(\mathrm{~s}, 9 \mathrm{H})$, $0.20(\mathrm{~s}, 6 \mathrm{H}), 0.06(\mathrm{~s}, 6 \mathrm{H}) .{ }^{13} \mathrm{C}$ NMR (101 MHz, $\left.\mathrm{CDCl}_{3}\right): \delta 155.2,153.8,149.6,145.8,133.8$, 123.1, 117.0, 115.4, 104.3, 62.7, 55.4, 34.3, 33.0, 26.0, 25.7, 18.5, 18.3, -4.6, -5.2. HRMS (ESI+): $m / z(\mathrm{M}+\mathrm{H})^{+}$calculated for $\mathrm{C}_{25} \mathrm{H}_{44} \mathrm{O}_{3} \mathrm{NSi}_{2} 462.2860$, found 462.2875 .

3-Butyl-7-fluoroisoquinoline (4r). $60 \mathrm{mg}(91 \%)$. Colorless oil, $\mathrm{R}_{f}=0.10$ (EtOAc/Hexane 10:90). ${ }^{1} \mathrm{H}$ NMR (400 MHz, $\left.\mathrm{CDCl}_{3}\right): \delta 9.16(\mathrm{~s}, 1 \mathrm{H}), 7.76(\mathrm{dd}, J=9.0,5.2 \mathrm{~Hz}, 1 \mathrm{H}), 7.53(\mathrm{dt}, J$ $=14.9,7.4 \mathrm{~Hz}, 1 \mathrm{H}), 7.47(\mathrm{~s}, 1 \mathrm{H}), 7.43(\mathrm{td}, J=8.8,2.6 \mathrm{~Hz}, 1 \mathrm{H}), 3.00-2.87(\mathrm{~m}, 2 \mathrm{H}), 1.88-1.71$ $(\mathrm{m}, 2 \mathrm{H}), 1.49-1.33(\mathrm{~m}, 2 \mathrm{H}), 0.96(\mathrm{t}, J=7.4 \mathrm{~Hz}, 3 \mathrm{H}) .{ }^{13} \mathrm{C} \mathrm{NMR}\left(126 \mathrm{MHz}, \mathrm{CDCl}_{3}\right): \delta 159.8$ $(\mathrm{d}, J=248 \mathrm{~Hz}), 155.5(\mathrm{~d}, J=1.6 \mathrm{~Hz}), 151.2(\mathrm{~d}, J=5.3 \mathrm{~Hz}), 133.5,128.7(\mathrm{~d}, J=8.3 \mathrm{~Hz})$, $127.4(\mathrm{~d}, J=8.0 \mathrm{~Hz}), 120.9(\mathrm{~d}, J=25.6 \mathrm{~Hz}), 117.7,110.4(\mathrm{~d}, J=20.5 \mathrm{~Hz}), 37.7,32.1,22.5$, 14.0. HRMS (ESI+): $m / z(\mathrm{M}+\mathrm{H})^{+}$calculated for $\mathrm{C}_{13} \mathrm{H}_{15} \mathrm{FN} 204.1188$, found 204.1191.

3,4-Dipropylisoquinoline (4s). ${ }^{19} 60 \mathrm{mg}$ (91\%). Colorless oil, $\mathrm{R}_{f}=0.40$ (EtOAc/Hexane 10:90). ${ }^{1} \mathrm{H}$ NMR $\left(400 \mathrm{MHz}, \mathrm{CDCl}_{3}\right): \delta 9.08(\mathrm{~s}, 1 \mathrm{H}), 7.97(\mathrm{dd}, J=8.6,0.7 \mathrm{~Hz}, 1 \mathrm{H}), 7.91(\mathrm{~d}, J$ $=8.1 \mathrm{~Hz}, 1 \mathrm{H}), 7.70-7.63(\mathrm{~m}, 1 \mathrm{H}), 7.51(\mathrm{ddd}, J=8.0,5.1,1.0 \mathrm{~Hz}, 1 \mathrm{H}), 3.05-2.99(\mathrm{~m}, 2 \mathrm{H})$, 2.99-2.92 (m, 2H), 1.86-1.78 (m, 2H), 1.73-1.65 (m, 2H), $1.10(\mathrm{t}, J=7.4 \mathrm{~Hz}, 3 \mathrm{H}), 1.05(\mathrm{t}, J=$ $7.4 \mathrm{~Hz}, 3 \mathrm{H}) .{ }^{13} \mathrm{C} \mathrm{NMR}\left(101 \mathrm{MHz}, \mathrm{CDCl}_{3}\right): \delta 152.9,150.1,135.3,129.9,128.1,128.0,127.2$, 125.6, 123.0, 37.3, 29.9, 224.1, 23.6, 14.6, 14.3. HRMS (ESI+): $m / z(\mathrm{M}+\mathrm{H})^{+}$calculated for $\mathrm{C}_{15} \mathrm{H}_{20} \mathrm{~N} 214.1596$, found 214.1601.

3-Butyl-1-methoxyisoquinoline (4t). $58 \mathrm{mg}$ (87\%). Colorless oil, $\mathrm{R}_{f}=0.10$ (EtOAc/Hexane 5:95). ${ }^{1} \mathrm{H}$ NMR (400 MHz, $\left.\mathrm{CDCl}_{3}\right): \delta 8.21-8.13(\mathrm{~m}, 1 \mathrm{H}), 7.64(\mathrm{~d}, J=8.1 \mathrm{~Hz}$, 1H), 7.58 (ddd, $J=8.2,6.8,1.3 \mathrm{~Hz}, 1 \mathrm{H}), 7.43$ (ddd, $J=8.2,6.8,1.3 \mathrm{~Hz}, 1 \mathrm{H}), 7.00(\mathrm{~s}, 1 \mathrm{H})$, $4.11(\mathrm{~s}, 3 \mathrm{H}), 2.82-2.73(\mathrm{~m}, 2 \mathrm{H}), 1.83-1.71(\mathrm{~m}, 2 \mathrm{H}), 1.46-1.36(\mathrm{~m}, 2 \mathrm{H}), 0.96(\mathrm{t}, J=7.4 \mathrm{~Hz}$, $3 \mathrm{H}) .{ }^{13} \mathrm{C} \mathrm{NMR}\left(126 \mathrm{MHz}, \mathrm{CDCl}_{3}\right): \delta 160.2,153.0,138.6,130.1,125.6,125.4,124.0,118.1$, 
111.7, 53.4, 37.6, 31.4, 22.4, 14.0. HRMS (ESI+): $m / z(\mathrm{M}+\mathrm{H})^{+}$calculated for $\mathrm{C}_{14} \mathrm{H}_{18} \mathrm{ON}$ 216.1388 , found 216.1398 .

3-Butyl-1-methylisoquinoline $\quad(\mathbf{4 u}){ }^{20} \quad 58 \mathrm{mg}$ (88\%). Colorless oil, $\mathrm{R}_{f}=0.10$ (EtOAc/Hexane 10:90). ${ }^{1} \mathrm{H} \mathrm{NMR}\left(400 \mathrm{MHz}, \mathrm{CDCl}_{3}\right): \delta 8.07(\mathrm{dd}, J=8.4,0.9 \mathrm{~Hz}, 1 \mathrm{H}), 7.73$ $(\mathrm{d}, J=8.2 \mathrm{~Hz}, 1 \mathrm{H}), 7.62(\mathrm{ddd}, J=8.2,6.8,1.2 \mathrm{~Hz}, 1 \mathrm{H}), 7.51$ (ddd, $J=8.2,6.8,1.3 \mathrm{~Hz}, 1 \mathrm{H})$, $7.32(\mathrm{~s}, 1 \mathrm{H}), 2.95(\mathrm{~s}, 3 \mathrm{H}), 2.92-2.86(\mathrm{~m}, 2 \mathrm{H}), 1.82-1.74(\mathrm{~m}, 2 \mathrm{H}), 1.48-1.39(\mathrm{~m}, 2 \mathrm{H}), 0.96(\mathrm{t}, J$ $=7.4 \mathrm{~Hz}, 3 \mathrm{H}) .{ }^{13} \mathrm{C} \mathrm{NMR}\left(126 \mathrm{MHz}, \mathrm{CDCl}_{3}\right): \delta 158.0,154.5,136.6,129.8,126.8,126.0$, 125.8, 125.5, 116.5, 37.9, 32.1, 22.6, 22.3, 14.0. HRMS $(\mathrm{ESI}+): \mathrm{m} / z(\mathrm{M}+\mathrm{H})^{+}$calculated for $\mathrm{C}_{14} \mathrm{H}_{18} \mathrm{~N} 200.1439$, found 200.1445.

6-Butylthieno [3,4-c] pyridine (8a). $56 \mathrm{mg}(86 \%)$. Colorless oil, $\mathrm{R}_{f}=0.40$ (EtOAc/Hexane 10:90). ${ }^{1} \mathrm{H}$ NMR (400 MHz, $\left.\mathrm{CDCl}_{3}\right): \delta 9.07$ (s, 1H), 7.67 (d, $\left.J=5.4 \mathrm{~Hz}, 1 \mathrm{H}\right), 7.55$ (s, 1H), $7.30(\mathrm{~d}, J=5.3 \mathrm{~Hz}, 1 \mathrm{H}), 2.94-2.87(\mathrm{~m}, 2 \mathrm{H}), 1.77(\mathrm{tt}, J=7.7,6.8 \mathrm{~Hz}, 2 \mathrm{H}), 1.47-1.35(\mathrm{~m}, 2 \mathrm{H})$, $0.96(\mathrm{t}, J=7.4 \mathrm{~Hz}, 3 \mathrm{H}) .{ }^{13} \mathrm{C} \mathrm{NMR}\left(101 \mathrm{MHz}, \mathrm{CDCl}_{3}\right): \delta 156.0,145.7,143.9,133.8,132.0$, 122.7, $116.2,37.7,32.5,22.5,14.0$. HRMS (ESI+): $\mathrm{m} / z(\mathrm{M}+\mathrm{H})^{+}$calculated for $\mathrm{C}_{11} \mathrm{H}_{14} \mathrm{NS}$ 192.0847, found 192.0856 .

6-(Cyclohexylmethyl)thieno[3,4-c]pyridine (8b). $63 \mathrm{mg}$ (94\%). Colorless oil, $\mathrm{R}_{f}=0.40$ (EtOAc/Hexane 10:90). ${ }^{1} \mathrm{H}$ NMR (400 MHz, $\left.\mathrm{CDCl}_{3}\right): \delta 9.08(\mathrm{~s}, 1 \mathrm{H}), 7.66(\mathrm{~d}, J=5.4 \mathrm{~Hz}$, $1 \mathrm{H}), 7.50(\mathrm{~d}, J=0.7 \mathrm{~Hz}, 1 \mathrm{H}), 7.29(\mathrm{dd}, J=5.4,0.7 \mathrm{~Hz}, 1 \mathrm{H}), 2.77(\mathrm{~d}, J=7.1 \mathrm{~Hz}, 2 \mathrm{H}), 1.85-$ $1.76(\mathrm{~m}, 1 \mathrm{H}), 1.70-1.62(\mathrm{~m}, 5 \mathrm{H}), 1.28-1.13(\mathrm{~m}, 3 \mathrm{H}), 1.09-0.95(\mathrm{~m}, 2 \mathrm{H}) .{ }^{13} \mathrm{C} \mathrm{NMR}(101 \mathrm{MHz}$, $\left.\mathrm{CDCl}_{3}\right): \delta 154.7,145.4,143.9,133.8,131.9,122.7,117.1,46.0,38.8,33.2,26.5,26.3$. HRMS (ESI+): $m / z(\mathrm{M}+\mathrm{H})^{+}$calculated for $\mathrm{C}_{14} \mathrm{H}_{18} \mathrm{NS} 232.1160$, found 232.1163 .

6-Benzylthieno[3,4-c]pyridine (8c). $59 \mathrm{mg}$ (88\%). Colorless oil, $\mathrm{R}_{f}=0.30$ (EtOAc/Hexane 20:80). ${ }^{1} \mathrm{H}$ NMR (500 MHz, $\left.\mathrm{CDCl}_{3}\right): \delta 9.09$ (s, 1H), 7.66 (d, $\left.J=5.3 \mathrm{~Hz}, 1 \mathrm{H}\right), 7.49$ (s, 1H), 7.31-7.29 (m, 4H), $7.25(\mathrm{~d}, J=2.8 \mathrm{~Hz}, 1 \mathrm{H}), 7.24-7.19(\mathrm{~m}, 1 \mathrm{H}), 4.28(\mathrm{~s}, 2 \mathrm{H}) .{ }^{13} \mathrm{C} \mathrm{NMR}(101$ $\left.\mathrm{MHz}, \mathrm{CDCl}_{3}\right): \delta 154.7,145.8,144.1,139.9,134.2,132.2,129.1,128.6,126.3,122.8,116.8$, 44.2. HRMS (ESI+): $\mathrm{m} / \mathrm{z}(\mathrm{M}+\mathrm{H})^{+}$calculated for $\mathrm{C}_{14} \mathrm{H}_{12} \mathrm{NS} 226.0690$, found 226.0686 .

3-Butylbenzo[4,5]thieno[2,3-c]pyridine (8d). $53 \mathrm{mg}$ (78\%), colourless oil, $\mathrm{R}_{f}=0.20$ (EtOAc/Hexane 10:90). ${ }^{1} \mathrm{H}$ NMR (500 MHz, $\left.\mathrm{CDCl}_{3}\right) \delta 9.05(\mathrm{~s}, 1 \mathrm{H}), 8.21(\mathrm{~d}, J=7.9 \mathrm{~Hz}, 1 \mathrm{H})$, $7.89(\mathrm{~d}, J=8.0 \mathrm{~Hz}, 1 \mathrm{H}), 7.85(\mathrm{~s}, 1 \mathrm{H}), 7.63-7.53(\mathrm{~m}, 1 \mathrm{H}), 7.50(\mathrm{t}, J=7.4 \mathrm{~Hz}, 1 \mathrm{H}), 3.07-2.86$ $(\mathrm{m}, 2 \mathrm{H}), 1.92-1.73(\mathrm{~m}, 2 \mathrm{H}), 1.52-1.39(\mathrm{~m}, 2 \mathrm{H}), 0.98(\mathrm{t}, J=7.4 \mathrm{~Hz}, 3 \mathrm{H}) .{ }^{13} \mathrm{C} \mathrm{NMR}(101 \mathrm{MHz}$, $\left.\mathrm{CDCl}_{3}\right): \delta 157.1,143.9,142.5,141.4,133.8,133.2,128.9,124.7,123.3,122.8,114.4,38.0$, 32.5, 22.5, 14.0. HRMS (ESI+): $\mathrm{m} / \mathrm{z}(\mathrm{M}+\mathrm{H})^{+}$calculated for $\mathrm{C}_{15} \mathrm{H}_{16} \mathrm{NS} 242,1004$, found 242.1015 . 
Synthesis of Quinisocaine 12: Compounds 10, 11 and 12 were prepared according to the above procedures (for compound 11: the reaction was performed at $40{ }^{\circ} \mathrm{C}$ for $18 \mathrm{~h}$ ).

(E)-2-(Dimethylamino)ethyl2-(2-(4,4,5,5-tetramethyl-1,3,2-dioxaborolan-2-yl)hex-1enyl)benzoate (10). From $0.89 \mathrm{mmol}$ of $9,{ }^{21} 221 \mathrm{mg}(62 \%)$. Colorless oil, $\mathrm{R}_{f}=0.50$ $\left(\mathrm{MeOH} / \mathrm{CHCl}_{3} 10: 90\right) .{ }^{1} \mathrm{H} \mathrm{NMR}\left(500 \mathrm{MHz}, \mathrm{CDCl}_{3}\right): \delta 7.92(\mathrm{dd}, J=7.8,0.9 \mathrm{~Hz}, 1 \mathrm{H}), 7.44(\mathrm{~s}$, 1H), 7.39-7.34 (m, 1H), 7.32 (dd, $J=9.6,2.7 \mathrm{~Hz}, 1 \mathrm{H}), 7.29-7.25(\mathrm{~m}, 1 \mathrm{H}), 4.38$ (t, $J=6.0 \mathrm{~Hz}$, 2H), $2.71(\mathrm{dd}, J=11.1,5.1 \mathrm{~Hz}, 2 \mathrm{H}), 2.35-2.29(\mathrm{~m}, 8 \mathrm{H}), 1.53-1.46(\mathrm{~m}, 2 \mathrm{H}), 1.43-1.35$ (m, $2 \mathrm{H}), 1.14(\mathrm{~s}, 12 \mathrm{H}), 0.93(\mathrm{t}, J=7.3 \mathrm{~Hz}, 3 \mathrm{H}) .{ }^{13} \mathrm{C} \mathrm{NMR}\left(101 \mathrm{MHz}, \mathrm{CDCl}_{3}\right): \delta$ 166.0, 140.4, 139.4, 130.3, 129.8, 129.2, 128.6, 127.4, 125.7, 82.1, 61.6, 56.7, 44.7, 36.2, 30.9, 23.6, 21.5, 13.0 (the carbon $\alpha$ to boron was not found). HRMS (ESI+): $m / z(\mathrm{M}+\mathrm{H})^{+}$calculated for $\mathrm{C}_{23} \mathrm{H}_{37} \mathrm{O}_{4} \mathrm{BN}$ 402.2816, found 402.2827.

(E) and (Z) 2-(Dimethylamino)ethyl 2-(2-azidohex-1-enyl)benzoate (11). From $0.5 \mathrm{mmol}$ of 10, $81 \mathrm{mg}(52 \%)$. Colorless oil, $\mathrm{R}_{f}=0.45\left(\mathrm{MeOH} \mathrm{CHCl}_{3} 10: 90\right){ }^{1} \mathrm{H}$ NMR $(500 \mathrm{MHz}$, $\left.\mathrm{CDCl}_{3}\right): \delta 7.89(\mathrm{dd}, J=7.8,0.9 \mathrm{~Hz}, 1 \mathrm{H}), 7.73(\mathrm{~d}, J=7.8 \mathrm{~Hz}, 1 \mathrm{H}), 7.54-7.41(\mathrm{~m}, 1 \mathrm{H}), 7.26-$ $7.22(\mathrm{~m}, 1 \mathrm{H}), 6.34(\mathrm{~s}, 1 \mathrm{H}), 4.41(\mathrm{t}, J=5.8 \mathrm{~Hz}, 2 \mathrm{H}), 2.75(\mathrm{t}, J=4.9 \mathrm{~Hz}, 2 \mathrm{H}), 2.56-2.40(\mathrm{~m}$, 2H), 2.37 (s, 6H), 1.73-1.58 (m, 2H), 1.53-1.40 (m, 2H), 0.99 (t, $J=7.3 \mathrm{~Hz}, 3 \mathrm{H}) .{ }^{13} \mathrm{C}$ NMR $\left(126 \mathrm{MHz}, \mathrm{CDCl}_{3}\right): \delta 167.2,136.5,136.3,131.5,130.7,130.3,128.7,126.5,114.0,62.5$, 57.6, 45.6, 33.5, 30.0, 22.1, 13.8. HRMS (ESI+): $\mathrm{m} / \mathrm{z}(\mathrm{M}+\mathrm{H})^{+}$calculated for $\mathrm{C}_{17} \mathrm{H}_{25} \mathrm{O}_{2} \mathrm{~N}_{4}$ 317.1977, found 317.1991.

Quinisocaine 12. From $0.16 \mathrm{mmol}$ of $11,34 \mathrm{mg}(81 \%)$. Colorless oil, $\mathrm{R}_{f}=0.16$ $\left(\mathrm{MeOH} / \mathrm{CHCl}_{3}\right.$ 10:90). ${ }^{1} \mathrm{H}$ NMR $\left(500 \mathrm{MHz}, \mathrm{CDCl}_{3}\right): \delta 8.19(\mathrm{~d}, J=8.3 \mathrm{~Hz}, 1 \mathrm{H}), 7.63(\mathrm{~d}, J=$ $8.1 \mathrm{~Hz}, 1 \mathrm{H}), 7.58$ (ddd, $J=8.1,6.8,1.2 \mathrm{~Hz}, 1 \mathrm{H}), 7.42$ (ddd, $J=8.1,6.8,1.2 \mathrm{~Hz}, 1 \mathrm{H}), 7.00$ (s, $1 \mathrm{H}), 4.67(\mathrm{t}, J=5.8 \mathrm{~Hz}, 2 \mathrm{H}), 2.87(\mathrm{t}, J=5.8 \mathrm{~Hz}, 2 \mathrm{H}), 2.80-2.70(\mathrm{~m}, 2 \mathrm{H}), 2.42(\mathrm{~s}, 6 \mathrm{H}), 1.77-$ $1.74(\mathrm{~m}, 2 \mathrm{H}), 1.46-1.33(\mathrm{~m}, 2 \mathrm{H}), 0.95$ (, $J=7.4 \mathrm{~Hz} \mathrm{t}, 3 \mathrm{H}) .{ }^{13} \mathrm{C} \mathrm{NMR}\left(100 \mathrm{MHz}, \mathrm{CDCl}_{3}\right): \delta$ $159.5,152.9,138.6,130.1,125.5,125.3,124.0,118.0,111.8,63.7,58.0,45.8,37.5,31.4$, 22.3, 14.0. HRMS (ESI+): $m / z(\mathrm{M}+\mathrm{H})^{+}$calculated for $\mathrm{C}_{17} \mathrm{H}_{25} \mathrm{ON}_{2} 273.1966$, found 273.1965.

Supporting Information. ${ }^{1} \mathrm{H}$ and ${ }^{13} \mathrm{C}$ spectra of compounds $1 \mathrm{~d}, \mathbf{2 c}, \mathbf{2 f}-\mathbf{k}, \mathbf{2 m}, \mathbf{2 p - 2 v}, \mathbf{3 a}-\mathbf{3 v}$, 4a-4u, 6a-6d, 7a-7d, 8a-8d, 10, 11 and quinisocaine 12. This material is available free of charge via the Internet at http://pubs.acs.org.

\section{Notes}


The authors declare no competing financial interest.

\section{ACKNOWLEDGMENTS}

This research has been performed as part of the Indo-French "Joint Laboratory for Sustainable Chemistry at Interfaces", FB and BC thank CNRS and the University of Rennes 1 for support of this research. V.J. and G.V.M.S thank UGC and CSIR, New Delhi for research fellowship.

\section{REFERENCES}

(1) a) Bentley, K. W. The isoquinoline alkaloids; Harwood Academic: Amsterdam, 1998. Khan, A. Y.; Kumar, G. S. Biophys Rev. 2015, 7, 407-420. b) Dembitsky, V. M.; Gloriozova, T. A.; Poroikov, V. V. Phytomedicine 2015, 22, 183-202. c) Bhadra, K.; Kumar, G. S. Med. Res. Rev. 2011, 31, 821-862.

(2) For some recent examples, see: (a) Zhao, Y.; Jing, Z.; Lv, J.; Zhang, Z.; Lin, J.; Cao, X.; Zhao, Z.; Liu, P.; Mao, W Biomed. Pharmacother. 2017, 95, 18-24. (b) Teodori, E.; Dei, S.; Bartolucci, G.; Perrone, M. G.; Manetti, D.; Romanelli, M. Novella; Contino, M.; Colabufo, N. A. ChemMedChem 2017, 12, 1369-1379. (c) Strong, K. L.; Epplin, M. P.; Bacsa, J.; Butch, C. J.; Burger, P. B.; Menaldino, D. S.; Traynelis, S. F.; Liotta, D. C. J. Med. Chem. 2017, 60, 5556-5585. d) Burks, H. E.; Abrams, T.; Kirby, C.A.; Baird, J.; Fekete, A.; Hamann, L. G.; Kim, S.; Lombardo, F.; Loo, A.; Lubicka, D.; Macchi, K.; McDonnell, D. P.; Mishina, Y.; Norris, J. D.; Nunez, J.; Saran, C.; Sun, Y.; Thomsen, N. M.; Wang, C.; Wang, J.; Peukert, S. J. Med. Chem. 2017, 60, 2790-2818. e) Bruno, E.; Buemi, M. R.; Di Fiore, A.; De Luca, L.; Ferro, S.; Angeli, A.; Cirilli, R.; Sadutto, D.; Alterio, V.; Monti, S. M.; Supuran, C. T.; De Simone, G, Gitto, R.et J. Med. Chem. 2017, 60, 4316-4326.

(3) For some recent examples, see: a) Han, H.-B.; Cui, R.-Z.; Jing, Y.-M.; Lu, G.-Z.; Zheng, Y.-X.; Zhou, L.; Zuo, J.-L.; Zhang, H. J. Mat. Chem. C: 2017, 5, 8150-8159. b) Tehfe, M.-A.; Lepeltier, M.; Dumur, F.; Gigmes, D.; Fouassier, J.-P.; Lalevee, J. Macromol. Chem. Phys. 2017, DOI: 10.1002/macp.201700397. c) Golden, J. H.; Facendola, J. W.; Sylvinson, M. R. D.; Baez, C. Q.; Djurovich, P. I.; Thompson, M. E. J. Org. Chem. 2017, 82, 7215-7222. d) Boynton, A. N.; Marcelis, L.; McConnell, 
A. J.; Barton, J. K. Inorg. Chem. 2017, 56, 8381-8389. e) Possato, B.; Deflon, V. M.; Naal, Z.; Formiga, A. L. B.; Nikolaou, S. Dalton Trans. 2017, 46, 7926-7938.

(4) Heravi, M.M.; Khaghaninejad, S.; Mostofi, M. Adv. Heterocycl. Chem. 2014, 112, 150.

(5) Hudson, A. Pomeranz-Fritsch Reaction in Name Reactions in Heterocyclic Chemistry; Li, J. J., Corey, E. J., Eds.; Wiley \& Sons: Hoboken, NJ, 2005, pp 480 486. b) Gensler, W. J. Organic Reactions; Adams, R., Ed.; Wiley: New York, 1951, 6, 191-206.

(6) For recent reviews, see: a) Jacob, J.; Varghese, N.; Rasheed, S. P.; Agnihotri, S.; Sharma, V.; Wakode, S. World J Pharm Pharm Sci. 2016, 5, 1821- 1837. b) Zhang, B.; Studer A. Chem. Soc. Rev., 2015, 44, 3505--3521. c) He, R.; Huang, Z.; Zheng, Q.; Wang, C. Tetrahedron Lett. 2014, 55, 5705-5713. d) Chevallier, F.; Mongin, F. Topics Het. Chem. 2013, 31, 93-129.

(7) For recent and selected examples of metal-catalyzed process, see: Palladium: a) Qi, L.; Hu, K.; Yu, S.; Zhu, J.; Cheng, T.; Wang, X.; Chen, J.; Wu, H. Org. Lett. 2017, 19, 218-221. b) Yao, T.; Liu, T.; Zhang, C. Chem. Commun. 2017, 53, 2386-2389. c) Cobalt: Kuai, C.; Lianhui, W.; Li, B.; Yang, Z.; Cui, X. Org. Lett. 2017, 19, 21022105. d) Wang, H.; Lorion, M. M.; Ackermann, L. ACS Catal. 2017, 7, 3430-3433. Ruthenium: e) Feng, S.; Li, T.; Du, C.; Chen, P. Song, D.; Li, J.; Xie, X.; Xuegong, S. Chem. Commun., 2017, 53, 4585-4588. Silver: f) Yao, Q.; Zhou, X.; Zhang, X.; Wang, C.; Wang, P.; Li, M. Org. Biomol. Chem. 2017, 15, 957-971. Rhodium: g) Tang, J.; Li, S.; Liu, Z.; Zhao, Y.; She, Z.; Kadam, V. D.; Gao, G.; Lan, J.; You, J. Org. Lett. 2017, 19, 604-607. Copper: h) Wen, L.-R.; Dou, Q.; Wang, Y.-C.; Zhang, J.-W.; Guo, W.-S.; Li, M. J. Org. Chem. 2017, 82, 1428-1436 and references cited therein.

(8) For recent reviews on intramolecular aza-Wittig reactions, see: a) Huang D.; Yan, G. Adv. Synth. Catal. 2017, 359, 1600-1619. b) Hu, B.; DiMagno, S.G. Org. Biomol. Chem. 2015, 13, 3844-3855. c) Molina, P.; Tarraga, A.; Alfonso, M. Eur. J. Org. Chem. 2011, 4505-4518.

(9) a) Tsotinis, A.; Eleutheriades, A.; Hough, K. A.; Davidson, K.; Sugden, D. Bioorg. Chem. 2007, 35, 189-204. b) Guanti, G.; Riva, R. Tetrahedron: Asymm 2001, 12, 1185-1200. c) Grunewald, G. L.; Sall, D. J.; Monn, J. A J. Med. Chem. 1988, 31, 824d) Kennedy, M.; Moody, C. J.; Rees, C. W.; Vaquero, J. J. Chem. Soc., Perkin Trans. 1 1987, 1395-1398. e) Hickey, D. M. B.; MacKenzie, A. R.; Moody, C. J.; Rees, C.W. 
J. Chem. Soc., Perkin Trans. 1 1987, 921-926. f) Hickey, D. M. B.; MacKenzie, A. R.; Moody, C. J.; Rees, C. W. J. Chem. Soc., Chem. Commun. 1984, 776-777.

(10) a) Hemelaere, R.; Caijo, F.; Mauduit, M.; Carreaux, F.; Carboni, B. Eur. J. Org. Chem. 2014, 3328-3333. b) Yang, H.; Li, Y.; Jiang, M.; Wang, J.; Fu, H. Chem. Eur. J. 2011, 17, 5652-5660. c) Shi, F.; Waldo, J. P.; Chen, Y.; Larock, R. C. Org. Lett. 2008, 10, 2409-2412. d) Li, Y.; Goa, L.-X.; Han, F.-S. Chem. Eur. J. 2010, 10, 79697972. e) Tao, C.; Cui, X.; Li, J.; Liu, A.; Liu, L.; Guo, Q. Tetrahedron Lett. 2007, 48, 3525-3529.

(11) a) Jayaram, V.; Sridhar, T.; Sharma, G. V. M.; Berree, F.; Carboni, B. J. Org. Chem. 2017, 82, 1803-1811. b) Sridhar, T.; Berree, F.; Sharma, G. V. M.; Carboni, B. J. Org. Chem. 2014, 79, 783-789.

(12) (a) Ishiyama, T.;Matsuda, N.;Miyaura, N.; Suzuki, A. J. Am. Chem. Soc. 1993, 115, 11018-11019. (b) Ishiyama, T.; Matsuda, N.; Murata, M.; Ozawa, F.; Suzuki, A.; Miyaura, N. Organometallics 1996, 15, 713-720.

(13) For a review on the chemistry of thienopyridines, see: a) Litvinov, V. P.; Dotsenko, V. V.; Krivokolysko, S. G. Adv. in Het. Chem. 2007, 93, 117-178. b) Litvinov, V. P.; Dotsenko, V. V.; Krivokolysko, S.G. Russ. Chem. Bull., Int. Ed., 2005, 54, 864-904.

(14) For some selected references on biological activities of thieno [2,3-c] pyridines, see: a) Wang, M.; Fang, Y.; Gu, S.; Chen, F.; Zhu, Z.; Sun, X.; Zhu, J. Eur. J. Med. Chem. 2017, 132, 157-172. b) Harris, N.; Koppel, J.; Zsila, F.; Juhas, S.; Il'kova, G.; Kogan, F. Y.; Lahmy, O.; Wildbaum, G.; Karin, N.; Zhuk, R.; Gregoret, P. Inflamm. Res. 2016, 65, 285-294. c) Tani, N.; Juvonen, R. O.; Raunio, H.; Fashe, M.; Leppanen, J.; Zhao, B.; Tyndale, R. F.; Rahnasto-Rilla, M. Bioorg. Med. Chem. 2014, 22, 66556664. d) Rubel, E.; Simon, J.; Chen, X.; Chowdhury, S.; Herr, R. J.; Jiang, Q.; Owens, K. N.; Raible, D.; Johnson, G. PCT Int. Appl. 2014, WO 2014052914. e) Hassan, A. Y.; Mohamed, H. A. Asian. J. Chem. 2009, 21, 3947-3961. f) Cusack, K.; Allen, H.; Bischoff, A.; Clabbers, A.; Dixon, R.; Fix-Stenzel, S.; Friedman, M.; Gaumont, Y.; George, D.; Gordon, T.; Grongsaard, P.; Bernd, J. Bioorg. Med. Chem. Lett. 2009, 19, 1722-1725. g) Amegadzie, A. K.; Dao, Y.; Gardinier, K. M.; Garmene, D. J.; Green, S. J.; Hembre, E. J.; Lu, J.; Spinazze, P. G. PCT Int. Appl. 2007, WO2007146759.

(15) a) Gentry, C. L.; Lukas, R. J. J. Pharm. Exp. Ther. 2001, 299, 1038-1048. b) Nishizawa, Y.; Gusovsky, F.; Daly, J. W. Biochim. Biophys Act. Mol. Cell. Res. 1990, 1054, 213-218. c) Gupta, S. P.; Gupta, J. K.; Saha, R. N. Drug Des. Del. 1990, 6, 131135. d) Casanovas, A. M.; Labat, C.; Courriere, P.; Oustrin, J. Biochem. Pharm. 1985, 
34, 3187-90. e) Casanovas, A. M.; Labat, C.; Courriere, P.; Oustrin, J. Eur. J. Med. Chem. 1982, 17, 333-337. f) Lukas, R. J.; Bennett, E. L. FEBS Lett. 1979, 108, 356358.

(16) Gao, H.; Zhang, J. Adv. Synth. Catal. 2009, 351, 85-88.

(17) Dell'Acqua, M.; Pirovano, V.; Confalonieri, G.; Arcadi, A.; Rossi, E.; Abbiati, G Org. Biomol. Chem., 2014, 12, 8019-8030.

(18) Coppola, A.; Sucunza, D.; Burgos; C.; Vaquero, J. J. Org. Lett. 2015, 17, 78-81.

(19) Guimond, N.; Fagnou, K. J. Am. Chem. Soc. 2009, 131, 12050-12051.

(20) Zhao, X.; Fan, W.; Miao, Z.; Chen, R. Synth. Commun. 2013, 43, 1714-1720.

(21) Hu, Z.X.; An, Q.; Li, K.F.; Zhang, Y.G.; Qiu, J.Y.; Xu, B.X.; Pan, W.D.; Cao, P.X.; Liu, C.X.; Huang, Z.M.; Xia, W.; Liang, G.Y. Org. Process. Res. Dev. 2013, 17, 1156-1167. 